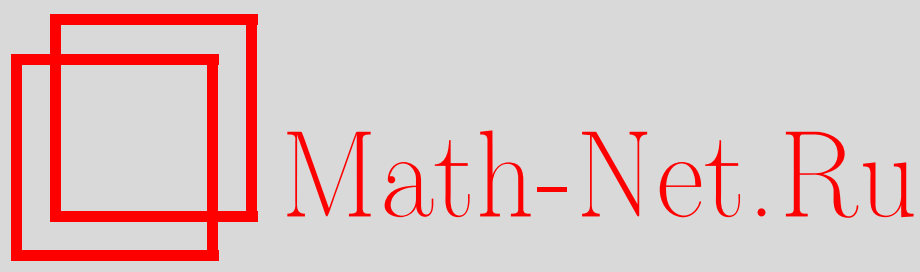

А. М. Гайсин, Оценки роста и убывания целой функции бесконечного порядка на кривых, Матем. сб., 2003, том 194, номер 8, 55-82

DOI: https://doi.org/10.4213/sm761

Использование Общероссийского математического портала Math-Net.Ru подразумевает, что вы прочитали и согласны с пользовательским соглашением http://www . mathnet.ru/rus/agreement

Параметры загрузки:

IP: 54.224 .187 .69

26 апреля 2023 г., 10:24:13 
УДК 517.53

\author{
А.М. Гайсин
}

\title{
Оценки роста и убывания целой функции бесконечного порядка на кривых
}

\footnotetext{
Изучается связь между ростом целого ряда Дирихле с лакунами Фейера и его убыванием на кривых, у ходящих в бесконечность.

В самой общей ситуации установлены неулучшаемые оценки.

Библиография: 32 названия.
}

В статье обсуждается одна классическая задача об асимптотическом поведении целых функций, представленных лакунарньпи степенными рядами.

Пусть $\left\{p_{n}\right\}$ - возрастающая последовательность натуральных чисел, удовлетворяющая условию

$$
\sum_{n=1}^{\infty} \frac{1}{p_{n}}<\infty
$$

В этом случае говорят, что последовательность $\left\{p_{n}\right\}$ имеет лакуныц Фейера. Аналогично, целая функция

$$
f(z)=\sum_{n=0}^{\infty} c_{n} z^{n}
$$

имеет лакуны Фейера, если последовательность $S(f)=\left\{n \geqslant 1: c_{n} \neq 0\right\}$ имеет лакуны Фейера. В этом случае ряд (2) есть лакунарный степенной ряд вида

$$
f(z)=c_{0}+\sum_{n=1}^{\infty} a_{n} z^{p_{n}}, \quad a_{n}=c_{p_{n}} \neq 0 .
$$

Хорошо известно, что целая функция с лакунами Фейера принимает каждое комплексное значение бесконечно много раз [1]. Этот замечательный факт и другие соображения наводят на мысль о наличии у целых функций $f(z)$, заданных рядами (3), хороших асимптотических свойств. Это подтверждается многочисленньми исследованиями, которые проводились специалистами по теории функций в течение многих лет. Имеется обширная литература по данной тематике, далеко не полный перечень которой мы приводим в конце работы (см., например, [1]-[27]). В подавляюшем большинстве этих работ в основном указаны достаточные условия, при выполнении которых справедливы утверждения:

а) при $r \rightarrow \infty$ вне некоторого исключительного множества $E \subset[0, \infty)$

$$
\ln M(r ; f)=(1+o(1)) \ln m(r ; f),
$$

Работа вьполнена при финансовой поддержке Российского фонда фундаментальных исследований (грант № 99-01-00655).

(C) А.М. ГАЙСИн 2003 
где

$$
M(r ; f)=\max _{|z|=r}|f(z)|, \quad m(r ; f)=\min _{|z|=r}|f(z)|
$$

или

b) для любой кривой $\gamma$, уходящей в бесконечность, существует последовательность $\left\{\xi_{n}\right\}, \xi_{n} \in \gamma$, такая, что при $\xi_{n} \rightarrow \infty$

$$
\ln M\left(\left|\xi_{n}\right| ; f\right)=(1+o(1)) \ln \left|f\left(\xi_{n}\right)\right|
$$

Впервые задача b) была сформулирована Полиа в работе [3] и решена для одного класса целых функций $f(z)$ вида $(3)$, имеющих конечный порядок. В случае, когда функция $f(z)$ имеет конечный порядок или конечный нижний порядок, в последние годы получены окончательные результаты [17]-[19] (в [18], [19] соответствующие результаты установлены для более общих рядов - рядов Дирихле). Когда же целая функция $f(z)$ (даже имеющая лакуны Фейера) имеет произвольньй рост, возникают существенные трудности, связанные с нерегулярньм распределением точек последовательности $\left\{p_{n}\right\}$, и поэтому полученные до сих пор результаты были далеки от окончательных.

Цель работы - дать полное решение задачи Пойа.

Мы здесь ограничимся задачами типа b). Утверждения же типа а), означающие более сильную регулярность роста функции $f(z)$, требуют отдельного рассмотрения.

Сделаем краткий обзор результатов, имеющих непосредственное отношение к обсуждаемым здесь задачам. Прежде всего отметим следующий факт, установленный в работе [2]: для того чтобы любая целая функция вида (3) не была ограниченной на луче $\mathbb{R}_{+}=[0, \infty)$, необходимо и достаточно, чтобы выполнялось условие (1). Аналогичное утверждение имеет место и для рядов Дирихле [27].

Пусть $L-$ класс всех непрерьвных на $[0, \infty)$ функций $w=w(x)$ таких, что $0<w(x) \uparrow \infty, x \rightarrow \infty$,

$$
W=\left\{w: w \in L, \int_{1}^{\infty} \frac{w(x)}{x^{2}} d x<\infty\right\}, \quad \Omega=\left\{w \in W: \frac{w(x)}{x} \downarrow 0, x \rightarrow \infty\right\}
$$

Последовательность $\left\{p_{n}\right\}$ назьвается интерполяционной, если найдется функция $w \in \Omega$, зависяшая только от последовательности $\left\{p_{n}\right\}$, такая, что для любой последовательности $\left\{b_{n}\right\} \subset \mathbb{C},\left|b_{n}\right| \leqslant 1$, существует целая функция экспоненциального типа $\varphi(z)$, обладающая свойствами:

$$
\varphi\left(p_{n}\right)=b_{n}, \quad n \geqslant 1, \quad \max _{|z|=r}|\varphi(z)|=M(r ; \varphi) \leqslant e^{w(r)}
$$

Это определение дано в работе [12], хотя интерполяционный метод был использован А.И. Павловым еще в работе [10], где он показал, что если

$$
\text { c) } \sum_{n=1}^{\infty} \frac{1}{p_{n}}<\infty, \quad \text { d) } \frac{n}{p_{n}} \downarrow
$$


TO

$$
d(f ; \gamma) \stackrel{\text { def }}{=} \varlimsup_{z \in \gamma} \frac{\ln |f(z)|}{z \rightarrow \infty}=1
$$

где $f(z)$ - целая функция, заданная рядом (3), а $\gamma$ - любая кривая, уходящая в бесконечность. Отметим, что в этой теореме равенство (5) получено без каких-либо ограничений на рост функции $f(z)$. Ранее равенство (5) было установлено Т. Ковари для последовательностей $\left\{p_{n}\right\}$ таких, что [6]

$$
p_{n}>n(\ln n)^{1+\varepsilon}, \quad n \geqslant n_{0}, \quad \varepsilon>0
$$

В [12] показано, что последовательности $\left\{p_{n}\right\}$, удовлетворяющие условиям (4), а также последовательности $\left\{p_{n}\right\}$, удовлетворяющие условию

$$
p_{n}>c n \ln n[\ln \ln n]^{2+\eta}, \quad n \geqslant n_{1}, \quad c>0, \quad \eta>0
$$

являются интерполяционными. Последовательности $\left\{p_{n}\right\}, p_{n}>c n \ln n[\ln \ln n]^{2}$, вообще говоря, не интерполяционные [13].

Таким образом, никак не связанные на первый взгляд условия Павлова и Ковари означают не что иное, как условие интерполяционности в смысле Коревара и Диксона.

Что же на самом далее означает условие интерполяционности?

В работе [13] установлен следующий критерий: для того чтобы последовательность $\left\{p_{n}\right\}$ была интерполяционной, необходимо и достаточно, чтобы существовала функция $w \in \Omega$ такая, что:

$$
\begin{aligned}
& \text { e) } n(t) \leqslant w(t), \quad n(t)=\sum_{p_{n}<t} 1 \\
& \text { f) }-\ln \prod_{\frac{p_{n}}{2}<p_{k}<2 p_{n}}\left|1-\frac{p_{n}}{p_{k}}\right| \leqslant w\left(p_{n}\right), \quad n \geqslant 1 .
\end{aligned}
$$

В статье [15] понятие интерполяционных последовательностей распространяется на произвольные вешественные последовательности $\left\{\lambda_{n}\right\}, 0<\lambda_{n} \uparrow \infty$, и для таких последовательностей аналог равенства (5) доказывается для более общих рядов - рядов Дирихле

$$
F(s)=\sum_{n=1}^{\infty} a_{n} e^{\lambda_{n} s}, \quad s=\sigma+i t
$$

сходящихся во всей плоскости.

Для интерполящионных последовательностей $\left\{\lambda_{n}\right\}$ автоматически выполняется условие [13], [15]

$$
\sum_{n=1}^{\infty} \frac{1}{\lambda_{n}}<\infty
$$

Однако для интерполяционности последовательности $\left\{\lambda_{n}\right\}$ только этого условия недостаточно [13]. При выполнении условия (7) ряды (6) будем называть рядами Дирихле с лакунами Фейера. 
Пусть $0<\lambda_{n} \uparrow \infty$,

$$
\sum_{n=1}^{\infty} \frac{1}{\lambda_{n}^{2}}<\infty, \quad Q(z)=\prod_{n=1}^{\infty}\left(1-\frac{z^{2}}{\lambda_{n}^{2}}\right), \quad q\left(\lambda_{n}\right)=-\ln \left|Q^{\prime}\left(\lambda_{n}\right)\right| .
$$

Введем функцию $\alpha(t)$, полагая $\alpha(t)=0$ при $0 \leqslant t<\lambda_{1}$ и $\alpha(t)=\max _{\lambda_{n} \leqslant t} q^{+}\left(\lambda_{n}\right)$ при $t \geqslant \lambda_{1}$, где $a^{+}=\max (a, 0)$.

Справедлива следуюшая теорема [26].

Теорема А. Пусть выполнено условие (7). Если

$$
\int_{1}^{\infty} \frac{\alpha(t)}{t^{2}} d t<\infty
$$

mo

$$
d(F ; \gamma) \stackrel{\text { def }}{=} \varlimsup_{\substack{s \in \gamma \\ s \rightarrow \infty}} \frac{\ln |F(s)|}{\ln M(\operatorname{Re} s)}=1
$$

где $M(\sigma)=\sup _{|t|<\infty}|F(\sigma+i t)|$, а $\gamma-$ любая кривая, уходящая в бесконечность maк, что если $s \in \gamma$ us $\rightarrow \infty$, mo $\operatorname{Re} s \rightarrow+\infty$.

Условия (7), (9) означают следующее: существует функция $w \in W$ такая, что

1) $n(t) \leqslant w(t)$,

2) $q\left(\lambda_{n}\right) \leqslant w\left(\lambda_{n}\right), n \geqslant 1$.

Эти же оценки следуют и из условий е), f) интерполяционности, но с функцией $w \in \Omega$. Так что условия теоремы А менее ограничительны, чем условия интерполяционности. Действительно, пусть $\Delta_{j}=\left[2^{j^{2}}-\left[\frac{2^{j^{2}}}{j^{2}}\right], 2^{j^{2}}\right]$ ([a]- целая часть $\left.a\right)$, а $\left\{\lambda_{n}\right\}$ - возрастаюшая последовательность всех натуральных чисел из $\bigcup_{j} \Delta_{j}$. В [23] показано, что для этой последовательности условия (7) и (9) выполнены, хотя она не интерполяционная. Видим, что условия теоремы А более слабые, чем условия интерполяционности. Но для справедливости теоремы А условие (7) необходимо: для любой последовательности $\left\{\lambda_{n}\right\}$, для которой

$$
\sum_{n=1}^{\infty} \frac{1}{\lambda_{n}}=\infty
$$

существует целая функция $F(s)$ вида $(6)$, для которой $d\left(F ; \mathbb{R}_{+}\right) \leqslant 0$, где $\mathbb{R}_{+}=$ $[0, \infty)[2],[27]$.

До настоящего времени оставался открытым следующий вопрос: каковы минимальные ограничения на последовательность $\left\{\lambda_{n}\right\}$, при которых для любой целой функции $F(s)$, заданной рядом (6) и имеюшей произвольньй рост, было бы справедливо равенство inf $\gamma \in \Gamma$ $d(F ; \gamma)=1$ ? Здесь $\Gamma=\{\gamma\}-$ семейство всех кривых $\gamma$, удовлетворяющих условиям теоремы А. Еще в работе [24] было высказано предположение о справедливости равенства $d\left(f ; \mathbb{R}_{+}\right)=1$ для любой целой функции с вешественными коэффициентами Тейлора, последовательность $\left\{p_{n}\right\}$ перемен знаков коэффициентов которой удовлетворяет лишь условию (1). В [24] было даже приведено доказательство этого сильного утверждения. Позднее обнаружилось, что 
в доказательстве есть серьезный пробел, который М. Н. Шеремета не смог устранить, и гипотезу из [24] он сформулировал как открытую проблему. До сих пор существовала аналогичная гипотеза М. Н. Шереметы о справедливости равенства $d\left(f ; \mathbb{R}_{+}\right)=1$ или более обшего равенства $\inf _{\gamma} d(f ; \gamma)=1$ для произвольных целых функций (3), имеющих лакуны Фейера. Однако до последнего времени не был известен ответ ни на одну из этих гипотез, формулировки которых в той или иной форме неоднократно приводились в разделах открытых проблем ряда выпусков журнала “Математичнї студіі” (Львов) последних лет. Следует отметить, что обе гипотезы по сути сводятся к одной и той же задаче из теории целых функций [28]. Положительньй ответ на последнюю гипотезу полностью разрешил бы проблему Макинтайра, в которой утверждается,что при условии (1) любая целая функция (3) не имеет конечных асимптотических значений. Но в работе [25] возникла новая гипотеза о том, что условие (9) теоремы А не может быть ослаблено: если интеграл (9) расходится, то сушествует целая функция $f(z)$ с лакунами Фейера, для которой $\inf _{\gamma} d(f ; \gamma)<1$. Однако построение соответствуюшего примера даже для какой-то специальной последовательности $\left\{p_{n}\right\}$ оказалось весьма нелегким делом.

В настоящей работе построен следующий пример: для любой последовательности $\left\{p_{n}\right\}$ с лакунами Фейера, но для которой интеграл (9) расходится, имеется функция $f(z)$ вида $(3)$ такая, что $d\left(f ; \mathbb{R}_{+}\right)=0$.

Основным результатом статьи является

ТЕОрема. Пусть $d(f)=\inf _{\gamma} d(f ; \gamma)$. Для того чтобъ для любой иелой функции $f$ вида (3) имело место равенство $d(f)=1$, необходимо и достаточно, чтобъ для последовательности $\left\{p_{n}\right\}$ выполнялись условия (1) и (9).

В обшей ситуации, т.е. когда вьполняется только условие (1), можно лишш утверждать, что $0 \leqslant d(f) \leqslant 1$ (см. следствие 2 из теоремы 2 ), причем обе оценки точные, а именно: для любой последовательности $\left\{p_{n}\right\}$ с лакунами Фейера, но для которой интеграл типа (9) расходится, имеются функции $f_{1}, f_{2}$, имеющие вид (3), для которых $d\left(f_{1}\right)=0, d\left(f_{2}\right)=1$.

Таким образом, в статье дается отрицательный ответ на гипотезу М.Н. Шереметы и получено окончательное решение задачи b) Полиа из [3].

\section{§1. Формулировки результатов}

Пусть $\Lambda=\left\{\lambda_{n}\right\}, 0<\lambda_{n} \uparrow \infty,-$ последовательность, удовлетворяющая условию

$$
\varlimsup_{n \rightarrow \infty} \frac{\ln n}{\ln \lambda_{n}}=a<\infty .
$$

Через $D(\Lambda)$ обозначим класс всех функций $F(s)$, представимых во всей плоскости рядами Дирихле

$$
F(s)=\sum_{n=1}^{\infty} a_{n} e^{\lambda_{n} s}, \quad s=\sigma+i t .
$$

Из условия (11) следует, что

$$
\varlimsup_{n \rightarrow \infty} \frac{\ln n}{\lambda_{n}}=0 .
$$


Так что ряд (12) сходится во всей плоскости абсолютно и равномерно, а его сумма $F(s)$ - целая функция [20]. Наряду с (12) введем в рассмотрение и ряд

$$
F^{*}(s)=\sum_{n=1}^{\infty} a_{n} b_{n} e^{\lambda_{n} s},
$$

где последовательность $b=\left\{b_{n}\right\}$ комплексных чисел $b_{n}\left(b_{n} \neq 0\right.$ при $\left.n \geqslant N\right)$ удовлетворяет условию

$$
\varlimsup_{n \rightarrow \infty} \frac{\ln \left|b_{n}\right|}{\lambda_{n}}<\infty .
$$

Тогда ряд (13) также сходится во всей плоскости, причем абсолютно и равномерно, а $F^{*}(s)$ - целая функция. В дальнейшем будем предполагать, что

$$
\varlimsup_{n \rightarrow \infty} \frac{1}{\lambda_{n}} \ln \left(\left|b_{n}\right|+\left|b_{n}\right|^{-1}\right)<\infty .
$$

Это позволит нам рассматривать также абсолютно сходящиеся во всей плоскости ряды Дирихле

$$
\sum_{n=N}^{\infty} b_{n}^{-1} a_{n} e^{\lambda_{n} s}
$$

Пусть $\mu(\sigma)$ и $\mu_{b}^{*}(\sigma)$ - максимальные члены рядов (12) и (13) соответственно, т.е.

$$
\mu(\sigma)=\max _{n \geqslant 1}\left\{\left|a_{n}\right| e^{\lambda_{n} \sigma}\right\}, \quad \mu_{b}^{*}(\sigma)=\max _{n \geqslant 1}\left\{\left|a_{n}\right|\left|b_{n}\right| e^{\lambda_{n} \sigma}\right\}
$$

Для нас особый интерес будет представлять случай, когда $b=\left\{Q^{\prime}\left(\lambda_{n}\right)\right\}$, где $Q-$ функция из (8). Но для упрощения записи далее вместо $\mu_{b}^{*}(\sigma)$ везде будем использовать символ $\mu^{*}(\sigma)$. Как и выше, $W$ будет означать так называемый класс сходимости, т.е. множество всех положительных непрерывных и неограниченно возрастаюших на $[0, \infty)$ функций $w=w(x)$ таких, что

$$
\int_{1}^{\infty} \frac{w(x)}{x^{2}} d x<\infty
$$

В дальнейшем считаем, что все исключительные множества, вне которых будут получены наши асимптотические оценки, представляют собой объединение конечного или бесконечного числа отрезков вида $\left[a_{n}, a_{n}^{\prime}\right]$, где $0<a_{1}<a_{1}^{\prime}<$ $a_{2}<a_{2}^{\prime}<\cdots<a_{n}<a_{n}^{\prime} \cdots$. При этом исключительное множество имеет конечную меру, если $\sum_{n}\left(a_{n}^{\prime}-a_{n}\right)<\infty$, и конечную логарифмическую меру, если $\sum_{n} \ln \left(a_{n}^{\prime} / a_{n}\right)<\infty$.

Имеет место следуюшая теорема.

ТЕОРЕМА 1. Пусть $\left\{b_{n}\right\}$ - некоторая последовательность комплексных чисел, удовлетворяющая условию $(14), \mu(\sigma)$ и $\mu^{*}(\sigma)$ - максимальнье члень рядов (12) и (13) соответственно.

Для того чтобъ для любой функиии $F \in D(\Lambda)$ при $\sigma \rightarrow+\infty$ вне некоторого множества $E \subset[0, \infty)$ конечной меры имело место асимптотическое равенство

$$
\ln \mu(\sigma)=(1+o(1)) \ln \mu^{*}(\sigma)
$$


необходимо и достаточно, чтобъ для некоторой функиии $w \in W$ выполнялись оченки

$$
\left|b_{n}\right|+\frac{1}{\left|b_{n}\right|} \leqslant e^{w\left(\lambda_{n}\right)}, \quad n \geqslant N
$$

Эта теорема, хотя представляет и самостоятельньй интерес, в случае $b=\left\{Q^{\prime}\left(\lambda_{n}\right)\right\}$ имеет весьма эффективное применение при доказательстве основной теоремы.

Пусть последовательность $\Lambda=\left\{\lambda_{n}\right\}$ имеет конечную верхнюю плотность $D$ и конечный индекс конденсации $\delta$, где

$$
D=\varlimsup_{n \rightarrow \infty} \frac{n}{\lambda_{n}}, \quad \delta=\varlimsup_{n \rightarrow \infty} \frac{1}{\lambda_{n}} \ln \frac{1}{\left|Q^{\prime}\left(\lambda_{n}\right)\right|} .
$$

Соответствующий класс целых функций $F(s)$, представимых во всей плоскости рядами Дирихле (12), будем обозначать через $B(\Lambda)$.

Отметим, что если, например, $\inf _{n}\left(\lambda_{n+1}-\lambda_{n}\right)=\tau>0$, то $\delta<\infty$ (см., напримep, [21]).

Рассмотрим следующий измененный ряд:

$$
F^{*}(s)=\sum_{n=1}^{\infty} a_{n} Q^{\prime}\left(\lambda_{n}\right) e^{\lambda_{n} s}
$$

где $a_{n}$ - коэффициенты ряда (12), а $Q(z)$ - целая функция, определенная формулой из (8). Если последовательность $\left\{\lambda_{n}\right\}$ имеет конечную верхнюю плотность, то $Q(z)$, а следовательно, и $Q^{\prime}(z)$, является целой функцией экспоненциального типа и ряд (17) сходится во всей плоскости абсолютно и равномерно. Отметим, что если вьполняется более сильное условие $(7)$, то функции $N(t)$ и $\ln M(t ; Q)$ принадлежат $W[21]$, где

$$
N(t)=\int_{0}^{t} \frac{n(x)}{x} d x, \quad n(x)=\sum_{\lambda_{j} \leqslant t} 1, \quad M(t ; Q)=\max _{|z|=t}|Q(z)| .
$$

Пусть $\Gamma=\{\gamma\}$ - семейство всех кривых $\gamma$, удовлетворяющих условиям теоремы $\mathrm{A}, \mu(\sigma)$ и $\mu^{*}(\sigma)$ - максимальные члены рядов (12) и (17) соответственно.

В этих обозначениях связь роста и убывания суммы ряда (12) устанавливается следуюшей теоремой.

ТЕОРема 2. Пусть выполняется условие $(7), \gamma \in \Gamma$. Тогда для любой функиии $F \in D(\Lambda)$ справедлива оценка

$$
q(F) \leqslant d(F)
$$

$3 \partial e c b$

$$
d(F)=\inf _{\gamma \in \Gamma} d(F ; \gamma), \quad q(F)=\inf _{e} \varlimsup_{\substack{\sigma \in e \\ \sigma \rightarrow \infty}} \frac{\ln \mu^{*}(\sigma)}{\ln \mu(\sigma)},
$$

$d(F ; \gamma)$ - величина, определенная формулой $(10)$, а точная нижняя грань и верхний предел в определении $q(F)$ вычисляются по всем множествам $е \subset \mathbb{R}_{+}$, кажсдое из которых получается удалением из $\mathbb{R}_{+}$некоторой системь отрезков конечной суммарной длинь. 
СЛЕДСТВИЕ 1. Пусть $\left\{b_{n}\right\}=\left\{Q^{\prime}\left(\lambda_{n}\right)\right\}$. Если асимптотическое равенство (15) имеет место при $\sigma \rightarrow \infty$ вне некоторого множества $E \subset \mathbb{R}_{+}$конечной мерь, то $q(F)=1$.

СлеДСтвИЕ 2. Для любой целой функиии $F \in D(\Lambda)$, имеющей лакуны Фейера, справедливы оченки

$$
0 \leqslant d(F) \leqslant 1
$$

əде $d(F)=\inf _{\gamma \in \Gamma} d(F ; \gamma)$.

Оценки (20) неулучшаемы. Действительно, с одной стороны, точность правой границы следует из теоремы А. С другой стороны, для любой последовательности $\left\{\lambda_{n}\right\}$, удовлетворяюшей условию (7) и имеющей конечный индекс конденсации, но для которой интеграл (9) расходится, существуют функции $F_{1} \in B(\Lambda), F_{2} \in B(\Lambda)$, для которых $q\left(F_{1}\right)=d\left(F_{1}\right)=1$, но $q\left(F_{2}\right)=d\left(F_{2} ; \mathbb{R}_{+}\right)=0$. Таким образом, смысл оценки (19) заключается в том, что величина $q(F)$ может равняться единице даже в том случае, когда условие (9) не выполняется. Соответствуюший пример построен в $\S 4$. Доказательство сушествования функции $F \in B(\Lambda)$, для которой $d\left(F ; \mathbb{R}_{+}\right)=0,-$ основной результат статьи. Этот факт устанавливается нами при доказательстве следующей теоремы.

Tеорема 3. Для того чтобъ для любой функиии $F \in B(\Lambda)$ с лакунами Фейера бъло справедливо равенство $d(F)=1$, необходимо и достаточно, чтобы выполнялось условие (9).

Из теорем 1-3 легко получаются аналогичные утверждения для целых функций, заданных степенными рядами (3). Чтобы убедиться в этом, необходимо сделать замену $z=e^{s}$. Только следует иметь в виду, что при такой замене исключительные множества $E \subset[0, \infty)$ конечной меры переходят на исключительные множества $e \subset[0, \infty)$ конечной логарифмической меры.

Оценки (20) устанавливают связь между ростом и убыванием целой функции $F(s)$ на каждой кривой $\gamma \in \Gamma$. Действительно, из оценки $0 \leqslant d(F ; \gamma)$ следует, что существуют функция $\varepsilon(r), \varepsilon(r) \downarrow 0$ при $r \rightarrow \infty$, и последовательность $\left\{\xi_{n}\right\}, \xi_{n} \in \gamma$, такие, что при $\xi_{n} \rightarrow \infty$

$$
\ln \left|F\left(\xi_{n}\right)\right|>-\varepsilon\left(\left|\left(\xi_{n}\right)\right|\right) \ln M\left(\operatorname{Re} \xi_{n}\right) .
$$

Отметим, что аналогичная оценка для произвольных целых функций на фиксированном луче была установлена Бёрлингом в работе [29]: для любых $\varepsilon>0$, $\theta \in[0,2 \pi)(\theta$ фиксировано) множество

$$
\left\{r \in \mathbb{R}_{+}: \ln \left|f\left(r e^{i \theta}\right)\right|>-(1+\varepsilon) \ln M(r ; f)\right\}
$$

неограниченное.

Хейманом в работе [30] рассмотрена аналогичная задача в случае, когда вместо луча берется произвольная кривая, уходящая в бесконечность. Он показал, что если нижний порядок целой функции $f(z)$ конечен, то на всякой кривой $\gamma$, уходящей в бесконечность, для любого $\varepsilon>0$ сушествует последовательность $\left\{z_{n}\right\}, z_{n} \in \gamma$, $z_{n} \rightarrow \infty$, такая, что [30]

$$
\ln \left|f\left(z_{n}\right)\right|>-(1+\varepsilon) \ln M\left(\left|z_{n}\right| ; f\right) .
$$


Там же утверждается, что эта оценка справедлива и для целых функций произвольного порядка.

Видим, что оценка (21) лучше соответствующих оценок Бёрлинга и Хеймана. Это объясняется тем, что в теореме 2 функция $F(s)$ имеет специальный вид, а именно является суммой ряда Дирихле с лакунами Фейера. Смысл оценки (21) в том, что при выполнении условия (7) сумма целого ряда Дирихле (12) не может сколь угодно быстро убывать на любой последовательности точек $\left\{\xi_{n}\right\}$, стремящейся к бесконечности вдоль кривой $\gamma$.

Таким образом, если из теоремы 2 вытекают неулучшаемые оценки для произвольных целых функций с лакунами Фейера на кривых, определенньм образом уходящих в бесконечность, то теорема 3 дает полный ответ на поставленную в работе [3] проблему.

\section{§2. Вспомогательные факты}

Сформулируем и докажем ряд вспомогательных утверждений, необходимых нам в дальнейшем.

В статье по существу будет использована следующая лемма типа Бореля-Неванлинны.

Лемма 1. Пусть $u \in L, w \in W, a v=v(\sigma)$-решение уравнения

$$
w(v)=e^{u(\sigma)} .
$$

Тогда при $\sigma \rightarrow \infty$ вне некоторого множества $E \subset[0, \infty)$ конечной мерь имеет место асимптотическое равенство

$$
u(\sigma+d h(\sigma))=u(\sigma)+o(1)
$$

әде $h=h(\sigma)=\frac{w(v(\sigma))}{v(\sigma)}$, a $d, 0<d<\infty,-$ фиксированная постоянная.

Лемма 1 доказана в работе [19].

Лемма 2. Пусть $f(z) \not \equiv 0$ - иелая функиия, а $\lambda>1$ - некоторая постоянная. Если $\sigma(r)$ - мера мнохсества тех точек $t$ из $(0, r)$, для которых

$$
\ln \min _{|z|=t}|f(z)|>-\lambda \ln M(r ; f)
$$

mo

$$
\lim _{r \rightarrow \infty} \frac{\sigma(r)}{r} \geqslant \frac{\lambda-1}{\lambda+1} .
$$

Лемма 2 есть следствие теоремы 3.5.6 из [31; гл. 3, §3.5]. Это следствие в [31] сформулировано неверно (правая часть $(22)$ вместо $M(r ; f)$ содержит величину $M(t ; f))$, на что обратил наше внимание А. Ю. Попов.

СлЕДСТВИЕ 3. Если $f(z)$ - челая функция вида (8), то существует последовательность $\left\{r_{n}\right\}, 0<r_{n} \uparrow \infty, r_{n-1}<r_{n} \leqslant 4 r_{n-1}, n \geqslant 2$, такая, что $\ln |f(z)|>-20 \ln M\left(r_{n} ; f\right),|z|=r_{n}$. 
ДоКАЗАТЕЛЬСТВо СЛЕДСТВИЯ 3. В лемме 2 положим $\lambda=5$. Тогда, применяя эту лемму к целой функции

$$
f(z)=\prod_{n=1}^{\infty}\left(1-\frac{z^{2}}{\lambda_{n}^{2}}\right)
$$

получаем, что

$$
\varliminf_{r \rightarrow \infty} \frac{\sigma(r)}{r} \geqslant \frac{2}{3}
$$

т.е. $\sigma(r)>r / 2$ при $r \geqslant 2^{n_{0}-1}$. Пусть $m(t ; f)=\min _{|z|=t}|f(z)|$. Тогда в каждом отрезке $\left[2^{n-1}, 2^{n}\right], n \geqslant n_{0}$, найдется точка $x_{n}$ такая, что

$$
\ln m\left(x_{n} ; f\right)>-5 \ln M\left(2^{n} ; f\right)>-5 \ln M\left(2 x_{n} ; f\right) .
$$

Но для нашей функции $f(z)$

$$
\ln M(r ; f)=2 r^{2} \int_{0}^{\infty} \frac{n(t)}{t\left(t^{2}+r^{2}\right)} d t, \quad n(t)=\sum_{\lambda_{j} \leqslant t} 1 .
$$

Следовательно,

$$
\ln m\left(x_{n} ; f\right)>-20 \cdot 2 x_{n}^{2} \int_{0}^{\infty} \frac{n(t)}{t\left[t^{2}+4 x_{n}^{2}\right]} d t>-20 \ln M\left(x_{n} ; f\right), \quad n \geqslant n_{0} .
$$

Поскольку $x_{n+1} \leqslant 2^{n+1}=4 \cdot 2^{n-1} \leqslant 4 x_{n}, n \geqslant n_{0}$, то последовательность $\left\{r_{n}\right\}$, $r_{n}=x_{n+n_{0}-1}, n \geqslant 1$, удовлетворяет всем требованиям.

TЕорема 4 (о двух константах). Пусть функция $f(z)$ аналитична в области $D$ и непрерывна в ее замыкании, а на граниче области $D$ для функиии $f(z)$ имеют место оценки

$$
|f(z)| \leqslant M, \quad z \in \partial D, \quad|f(z)| \leqslant m, \quad z \in E,
$$

где E - некоторое подмножество границы области $D$, а $m$ и $M, m \leqslant M$,некоторые положстельные постоянные. Тогда

$$
|F(z)| \leqslant m^{\gamma(z)} M^{1-\gamma(z)}, \quad z \in D,
$$

где $\gamma(z)=w(z ; E, D)$ - гармоническая мера множества $Е$ относительно области D.

Теорема 4 доказана в работе [31].

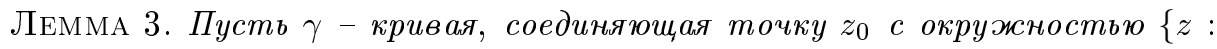
$\left.\left|z-z_{0}\right|=R\right\}$, состоящая из конечного числа кусочно-гладких жсордановых кривых, а $g(z)$ - функция, аналитическая в круге $D\left(z_{0} ; R\right)=\left\{z:\left|z-z_{0}\right|<R\right\}$ и непрерывная в его замыкании $\bar{D}\left(z_{0} ; R\right)$. Пусть

$$
M=\max _{\bar{D}\left(z_{0} ; R\right)}|g(t)|, \quad m=\max _{\gamma}|g(t)| .
$$

Тогда при $0<\beta \leqslant 1 / 5$ для всех $z$ из круга $\bar{D}\left(z_{0} ; \beta R\right)$ верна оценка:

$$
|g(z)| \leqslant m^{1-2 \beta} M^{2 \beta} .
$$


ДоКАЗАТЕЛЬСТво ЛЕмМЫ 3 основано на следуюшей теореме из [31]: пусть область $D$ представляет собой круг $D(0 ; R)$ с выброшенным из него множеством $E$, состоящим из конечного числа кусочно-гладких кривых и конечного числа областей, ограниченных такими кривыми, а $\Delta$ - некоторое подмножество интервала $(0, R)$, имеюшее меру $\delta$. Если для любого $x \in \Delta$ на окружности $|z|=x$ есть хотя бы одна точка, принадлежащая множеству $E$, то

$$
w(z ; E, D) \geqslant \frac{4}{\pi} \operatorname{arctg}\left[\frac{R-|z|}{R+|z|}\left(\frac{\delta}{2 R-\delta}\right)\right], \quad z \in D .
$$

Докажем лемму. Пусть для удобства $z_{0}=0$. Тогда, полагая $\Delta=(0, R), E=\gamma$, $D=D(0, R)$, из $(24)$ получаем, что если $|z| \leqslant \beta R, 0<\beta<1 / 2$, то

$$
w(z ; \gamma, D) \geqslant \frac{4}{\pi} \operatorname{arctg}(1-2 \beta)>0
$$

Рассмотрим функцию

$$
y(\beta)=\frac{4}{\pi} \operatorname{arctg}(1-2 \beta)-1+2 \beta .
$$

Имеем $y(0)=y(1 / 2)=0$. Проверяется, что при

$$
0<\beta<\beta_{1}=\frac{\pi-\sqrt{-\pi^{2}+4 \pi}}{2 \pi}
$$

функция $y=y(\beta)$ возрастает. Поскольку $1 / 5<\beta_{1}$, то $y(\beta)>0$ при $0<\beta \leqslant 1 / 5$. Следовательно, $w(z ; \gamma, D)>1-2 \beta$, если $|z| \leqslant \beta R, 0<\beta \leqslant 1 / 5$.

С учетом этой оценки, применяя теорему о двух константах, получаем требуемую оценку (23).

Лемма установлена.

ЛЕмма 4. Пусть $\rho(r)$ - уточненный порядок такой, что $\rho(r) \rightarrow 1$, $r^{1-\rho(r)} \rightarrow \infty$ при $r \rightarrow \infty$. Если $a_{n}, n \geqslant 1,-$ положсительнье числа, удовлетворяющие условию

$$
\frac{n}{a_{n}^{\rho\left(a_{n}\right)}}=\Delta, \quad 0<\Delta<\infty
$$

то иелая функиия

$$
g(z)=\prod_{n=1}^{\infty}\left(1+\frac{z^{2}}{a_{n}^{2}}\right)
$$

обладает свойствами:

1) при $\varphi \neq \pm \pi / 2$

$$
\lim _{r \rightarrow \infty} \frac{\ln \left|g\left(r e^{i \varphi}\right)\right|}{r^{\rho(r)}}=h(\varphi)=\pi \Delta|\cos \varphi|
$$

2) вне кружков $\left\{z:\left|z \pm a_{n} i\right|<1\right\}, n \geqslant 1$,

$$
\ln \left|g\left(r e^{i \varphi}\right)\right|>[h(\varphi)-\varepsilon] r^{\rho(r)}, \quad r>r_{0}(\varepsilon), \quad \forall \varepsilon>0 .
$$

Эта лемма есть частный случай теоремы 1.2.7 из [20; гл. I, §2] (см. там же замечание к теореме 1.2.5). 
Лемма 5. Пусть $\alpha(r)$ - положстельная неубьвающая и непрерывная на $[0, \infty)$ функиия порядка $\rho, 0<\rho<\infty$, принадлежсащая классу сходимости, m.e.

$$
\int_{1}^{\infty} \frac{\alpha(r)}{r^{2}} d r<\infty
$$

Тогда существует уточненный порядок $\rho(r)$ функиии $\alpha(r)$ такой, что

$$
\int_{1}^{\infty} r^{\rho(r)-\rho-1} d r<\infty .
$$

Лемма доказана в [32]. Она утверждает, что уточненный порядок $\rho(r)$ кроме (25) удовлетворяет условию

$$
\varlimsup_{r \rightarrow \infty} \frac{\alpha(r)}{r^{\rho(r)}}=1
$$

\section{§3. Максимальный член адамаровской композиции двух ря,дов Дирихле}

Пусть $F(s)$ - целая функция, определенная всюду сходящимся рядом Дирихле (12), показатели которого удовлетворяют условию

$$
\varlimsup_{r \rightarrow \infty} \frac{\ln n}{\lambda_{n}}=H<\infty .
$$

В силу этого условия ряд (12), поскольку он сходится во всей плоскости, сходится во всей плоскости абсолютно. Пусть $\mu(\sigma)$ - максимальный член, а $\nu=\nu(\sigma)-$ центральный индекс ряда (12), т.е.

$$
\mu(\sigma)=\max _{n \geqslant 1}\left\{\left|a_{n}\right| e^{\lambda_{n} \sigma}\right\}, \quad \nu=\nu(\sigma)=\max \left\{n \geqslant 1: \mu(\sigma)=\left|a_{n}\right| e^{\lambda_{n} \sigma}\right\}
$$

Отметим на плоскости точки $P_{n}=\left(\lambda_{n},-\ln \left|a_{n}\right|\right)$. Если для некоторого $n$ коэффиициент $a_{n}=0$, то будем считать, что ордината точки $P_{n}$ равна $+\infty$. Можно считать, что $a_{1} \neq 0$. Из сходимости ряда (12) во всей плоскости имеем

$$
\lim _{n \rightarrow \infty} \frac{\ln \left|a_{n}\right|}{\lambda_{n}}=-\infty .
$$

Учитывая это, через $Q(F)$ обозначим выпуклую оболочку точек $P_{n}, n \geqslant 1$. Пусть $\gamma(x)=\inf \{y:(x, y) \in Q(F)\}$. Выпуклая ломаная линия $L$, описываемая уравнением $y=\gamma(x), x \geqslant \lambda_{1}$, назьвается выпукльм полигоном Ньютона [20].

Ординату точки полигона Ньютона $L$ с абсциссой $\lambda_{n}$ обозначим через $G_{n}$. Для бесконечного множества значений $n$, в частности для $n=n_{i}, i=1,2, \ldots$, имеем $G_{n}=-\ln \left|a_{n}\right|$. Точка $P_{n}$ лежит либо на полигоне $L$ (точка $P_{n_{i}}$ обязательно лежит на полигоне), либо над ним.

Пусть точки $P_{n_{i}}$ с координатами $\left(\lambda_{n_{i}}, G_{n_{i}}\right)$ - вершины полигона $L$ (очевидно, $\left.n_{1}=1\right)$. Угловой коэффициент звена, соединяющего точки $P_{n_{i}}$ и $P_{n_{i+1}}$, равен

$$
R_{i}=\frac{G_{n_{i+1}}-G_{n_{i}}}{\lambda_{n_{i+1}}-\lambda_{n_{i}}}, \quad i \geqslant 1 .
$$


Ясно, что $R_{i} \uparrow \infty$ при $i \rightarrow \infty$. Следовательно [20; гл. II, $\S 6$, п. 2]:

1) при $R_{i-1} \leqslant \sigma<R_{i}$ центральный индекс $\nu(\sigma)=n_{i}=$ const и

$$
\ln \mu(\sigma)=\ln \left|a_{n_{i}}\right|+\lambda_{n_{i}} \sigma
$$

2) $\ln \mu(\sigma)$ - непрерывная функция; на участке $\left[R_{i-1}, R_{i}\right)$ она линейна, график ее на этом участке - прямая линия с угловым коэффициентом $\lambda_{n_{i}}$, причем $\lambda_{n_{i}} \uparrow \infty$ при $i \rightarrow \infty$. Следовательно, $\ln \mu(\sigma)$ - выпуклая возрастающая функция от $\sigma$.

Пусть $\Lambda=\left\{\lambda_{n}\right\}$ - последовательность, удовлетворяющая условию (11), $F \in$ $D(\Lambda)$, a

$$
G(s)=\sum_{n=1}^{\infty} b_{n} e^{\lambda_{n} s},
$$

где последовательность $\left\{b_{n}\right\}$ подчинена условию (14). Тогда ряд (26) абсолютно сходится в полуплоскости $\operatorname{Re} s<-b$. Адамаровская композиция рядов (12) и (26) есть ряд (13), т.е.

$$
(F * G)(s)=\sum_{n=1}^{\infty} a_{n} b_{n} e^{\lambda_{n} s}=F^{*}(s) .
$$

Ясно, что $F^{*} \in D(\Lambda)$. Как и вьшше, через $\mu(\sigma)$ обозначим максимальньй член ряда (12), через $\mu^{*}(\sigma)$ - максимальный член ряда (13).

ДОКАЗАТЕЛЬСТВО ТЕОРЕМЫ 1.

1. Достаточность. Пусть выполнено условие (16). Тогда сушествует функция $w^{*} \in W$ такая, что $\sqrt{x} \leqslant w^{*}(x)$ и $w(x)=o\left(w^{*}(x)\right)$ при $x \rightarrow \infty$. Пусть $v=v(\sigma)-$ решение уравнения

$$
w^{*}(v)=3 \ln \mu(\sigma) .
$$

Положим

$$
R_{v}=\sum_{\lambda_{j}>v}\left|a_{j}\right| e^{\lambda_{j} \sigma}, \quad h=\frac{w^{*}(v)}{v}, \quad v=v(\sigma) .
$$

Пусть $m \geqslant 1$ таково, что

$$
\sum_{n=1}^{\infty} \frac{1}{\lambda_{n}^{m}}<\infty
$$

В силу условия (11) такое $m$ сушествует. Тогда

$$
R_{v} \leqslant \mu(\sigma+h) \sum_{\lambda_{n}>v} e^{-h \lambda_{n}} \leqslant\left(\sum_{n=1}^{\infty} \frac{1}{\lambda_{n}^{m}}\right) \mu(\sigma+h) \exp \left[\max _{t \geqslant v} \varphi(t)\right],
$$

где $\varphi(t)=m \ln t-h t$. Поскольку $\varphi^{\prime}(t)=0$ в точке $t_{0}$,

$$
t_{0}=\frac{m}{h}=m \frac{v}{w^{*}(v)} \leqslant m \sqrt{v}<v=v(\sigma)
$$


при $\sigma \geqslant \sigma_{0}$, то

$$
R_{v} \leqslant C \mu(\sigma+h) \exp \{-v(1+o(1)) h\}
$$

при $\sigma \rightarrow \infty$. Отсюда, применяя лемму 1 , получаем, что при $\sigma \rightarrow \infty$ вне некоторого множества $E_{1} \subset[0, \infty)$ конечной меры

$$
R_{v} \leqslant C \mu(\sigma)^{1+o(1)} \exp \left\{-w^{*}(v)(1+o(1))\right\}=\mu(\sigma)^{-2(1+o(1))}, \quad C=\sum_{n=1}^{\infty} \frac{1}{\lambda_{n}^{m}} .
$$

Значит, при $\sigma \geqslant \sigma_{1}, \sigma \notin E_{1}$, получаем, что $\lambda_{\nu(\sigma)} \leqslant v(\sigma)$, где $\nu=\nu(\sigma)$ - центральный индекс ряда (12). Тогда

$$
\begin{aligned}
\mu(\sigma) & =\left|a_{\nu}\right| e^{\lambda_{\nu} \sigma}=\left|a_{\nu} b_{\nu}\right| e^{\lambda_{\nu} \sigma}\left|b_{\nu}\right|^{-1} \\
& \leqslant \mu^{*}(\sigma) e^{w(v)}=\mu^{*}(\sigma) \mu(\sigma)^{o(1)}, \quad \sigma \rightarrow \infty, \quad \sigma \notin E_{1} .
\end{aligned}
$$

Итак, при $\sigma \rightarrow \infty$ вне некоторого множества $E_{1} \subset[0, \infty)$ конечной меры

$$
(1+o(1)) \ln \mu(\sigma) \leqslant \ln \mu^{*}(\sigma) .
$$

Далее, поскольку $\left|b_{n}\right| \leqslant e^{w\left(\lambda_{n}\right)}, n \geqslant N$, то при $k \geqslant N$

$$
\mu^{*}(\sigma)=\left|a_{k} b_{k}\right| e^{\lambda_{k} \sigma} \leqslant \mu(\sigma) e^{w\left(\lambda_{k}\right)},
$$

где $k=k(\sigma)$ - центральный индекс ряда (13).

Пусть $x=x(\sigma)$ - решение уравнения $w^{*}(x)=3 \ln \mu^{*}(\sigma)$. Если

$$
R_{x}^{*}=\sum_{\lambda_{n}>x}\left|a_{n} b_{n}\right| e^{\lambda_{n} \sigma}
$$

то, применяя лемму 1 , получаем, что при $\sigma \rightarrow \infty$ вне некоторого множества $E_{2} \subset$ $[0, \infty)$ конечной меры

$$
R_{x}^{*} \leqslant C\left[\mu^{*}(\sigma)\right]^{-2(1+o(1))}, \quad C=\sum_{n=1}^{\infty} \frac{1}{\lambda_{n}^{m}} .
$$

Отсюда следует, что $\lambda_{k(\sigma)} \leqslant x(\sigma)$ при $\sigma \geqslant \sigma_{2}, \sigma \notin E_{2}$. Следовательно, из (28) получаем, что при $\sigma \rightarrow \infty$ вне $E_{2}$

$$
\mu^{*}(\sigma) \leqslant \mu(\sigma) e^{w(x(\sigma))}=\mu(\sigma)\left[\mu^{*}(\sigma)\right]^{o(1)},
$$

т.е.

$$
(1+o(1)) \ln \mu^{*}(\sigma) \leqslant \ln \mu(\sigma) .
$$

Из оценок $(27),(29)$ окончательно получаем, что при $\sigma \rightarrow \infty$ вне множества $E=E_{1} \cup E_{2}$, имеющего конечную меру,

$$
\ln \mu(\sigma)=(1+o(1)) \ln \mu^{*}(\sigma) \text {. }
$$

Достаточность доказана.

2. Необходимость. Условие (16) равносильно предложению: существует функция $\varphi \in W$ такая, что при $n \geqslant N$
1) $\left|b_{n}\right| \leqslant e^{\varphi\left(\lambda_{n}\right)}$
2) $\left|b_{n}\right|^{-1} \leqslant e^{\varphi\left(\lambda_{n}\right)}$. 
Действительно, то, что 1), 2) следуют из (16), очевидно. Обратно, из 1) и 2) имеем

$$
\left|b_{n}\right|+\left|b_{n}\right|^{-1} \leqslant 2 e^{\varphi\left(\lambda_{n}\right)}, \quad n \geqslant N .
$$

Следовательно, (16) имеет место с мажорантой $w(x)=\ln 2+\varphi(x)$. Так что если условие (16) не выполнено, то хотя бы для одной из последовательностей

$$
\left\{\ln \left|b_{n}\right|\right\}_{n=N}^{\infty}, \quad\left\{-\ln \left|b_{n}\right|\right\}_{n=N}^{\infty}
$$

не существует мажорантной последовательности вида $\left\{\varphi\left(\lambda_{n}\right)\right\}$, где $\varphi \in W$. Пусть для определенности последовательность $\left\{\ln \left|b_{n}\right|_{n=N}^{\infty}\right\}$ обладает этим свойством. Тогда

$$
\int_{\lambda_{N}}^{\infty} \frac{\alpha(t)}{t^{2}} d t=\infty
$$

где $\alpha(t)=\max _{\lambda_{n} \leqslant t}\left\{\ln \left|b\left(\lambda_{n}\right)\right|: n \geqslant N\right\}, b\left(\lambda_{n}\right)=b_{n}$. Не теряя общности, можем считать, что $\alpha(t)>0$ при $t \geqslant \lambda_{N}$.

Пусть $t_{0}=\lambda_{N}$, а $\left\{t_{n}\right\}_{n=1}^{\infty}-$ последовательность всех точек разрьва функции $\alpha(t)$. Положим $\gamma(t)=\sqrt{a(t)}$, где

$$
a(t)=\int_{\lambda_{N}}^{t} \frac{\alpha(x)}{x^{2}} d x .
$$

Ясно, что $0<a(t) \uparrow \infty$ при $t \rightarrow \infty$. Пусть $\alpha(t)=\alpha_{n}$ при $t_{n} \leqslant t<t_{n+1}, n \geqslant 0$, $\gamma_{s}=\gamma\left(t_{s}\right), s \geqslant 0$. Тогда

$$
\gamma_{s}^{2}=\int_{\lambda_{N}}^{t_{s}} \frac{\alpha(x)}{x^{2}} d x \leqslant \alpha_{s} \frac{1}{\lambda_{N}}
$$

Отсюда следует, что $\lambda_{N} \gamma_{s} \leqslant \alpha_{s} / \gamma_{s}$ и $\lim _{s \rightarrow \infty} \alpha_{s} / \gamma_{s}=\infty$. Следовательно, существует подпоследовательность $\left\{n_{j}\right\}$ такая, что

$$
\frac{\alpha\left(t_{n_{j}}\right)}{\gamma_{n_{j}}}<\frac{\alpha\left(t_{n_{j+1}}\right)}{\gamma_{n_{j+1}}}, \quad j \geqslant 1
$$

Положим

$$
\beta(t)=\left\{\begin{array}{l}
\beta_{1}=\gamma_{n_{1}}, \text { если } \lambda_{N} \leqslant t<t_{n_{1}}, \\
\beta_{j}=\gamma_{n_{j}}, \text { если } t_{n_{j}} \leqslant t<t_{n_{j}+1}, j \geqslant 1 .
\end{array}\right.
$$

Видим, что функция $\beta(t)$ положительная, неубывающая и $\beta(t) \rightarrow \infty$ при $t \rightarrow \infty$. Кроме того, множество точек разрыва функции $\beta(t)$ содержится в $\left\{t_{n}\right\}$. Отсюда, учитьвая (30), заключаем, что функция $g(t)=\alpha(t) \beta^{-1}(t)$ неубывающая и положительная на $\left[\lambda_{N}, \infty\right)$. Более того,

$$
I=\int_{\lambda_{N}}^{\infty} \frac{g(t)}{t^{2}} d t=\infty .
$$

Действительно, если $m_{j}=t_{n_{j}}$, то

$$
I\left(m_{j}\right)=\int_{\lambda_{N}}^{m_{j}} \frac{g(t)}{t^{2}} d t \geqslant \frac{a\left(m_{j}\right)}{\gamma\left(m_{j}\right)}=\sqrt{a\left(m_{j}\right)} .
$$


Следовательно, $I=\lim _{j \rightarrow \infty} I\left(m_{j}\right)=\infty$. Имея это в виду, рассмотрим последовательность $\left\{x_{n}\right\}, n \geqslant 0$, где

$$
x_{n}=\frac{G_{n+1}-G_{n}}{t_{n+1}-t_{n}}, \quad G_{n}=t_{n} I\left(t_{n}\right) .
$$

Проверяется, что

$$
x_{n}=I\left(t_{n}\right)+\frac{t_{n+1}}{t_{n+1}-t_{n}} \int_{t_{n}}^{t_{n+1}} \frac{g(t)}{t^{2}} d t .
$$

Значит, $x_{n} \rightarrow \infty$ при $n \rightarrow \infty$. Из неубывания функции $g(t)$ также следует, что $x_{n}<x_{n+1}, n \geqslant 0$. Итак, $0<x_{n} \uparrow \infty, n \rightarrow \infty$.

Пусть $y=\varphi_{n}(x)$ - уравнение прямой, проходяшей через точки $P_{n}=\left(t_{n} ; G_{n}\right)$ и $P_{n+1}=\left(t_{n+1} ; G_{n+1}\right)$. Поскольку $0<x_{n} \uparrow \infty, n \rightarrow \infty$, то последовательность $\left\{P_{n}\right\}$ есть вершины, а совокупность всех отрезков прямых $y=\varphi_{n}(x)$, соединяющих точки $P_{n}$ и $P_{n+1}, n \geqslant 0$, есть выпуклый полигон Ньютона $L$ для ряда Дирихле

$$
F(s)=\sum_{k=1}^{\infty} a_{k} e^{\lambda_{k} s}, \quad s=\sigma+i t
$$

где $a_{1}=a_{2}=\cdots=a_{N-1}=e^{-G_{1}}$, а для $k \geqslant N$

$$
a_{k}= \begin{cases}e^{-G_{n}}, & \text { если } k=j_{n}, \\ e^{-\varphi_{n}\left(\lambda_{k}\right)-1}, & \text { если } j_{n}<k<j_{n+1} .\end{cases}
$$

Здесь $\left\{j_{n}\right\}$ - последовательность индексов таких, что $t_{n}=\lambda_{j_{n}}, n \geqslant 0$. Заметим, что $t_{0}=\lambda_{N}$, а $\left\{t_{n}\right\}_{n=1}^{\infty}-$ последовательность центральных индексов ряда (31), абсолютно сходящегося во всей плоскости.

Оценим максимальный член $\mu(\sigma)$ ряда (31) сверху. Для $x_{n-1} \leqslant \sigma<x_{n}$ имеем

$$
\ln \mu(\sigma)=t_{n}\left(-I\left(t_{n}\right)+\sigma\right)<\frac{t_{n} t_{n+1}}{t_{n+1}-t_{n}} \int_{t_{n}}^{t_{n+1}} \frac{g(t)}{t^{2}} d t=\frac{\alpha_{n}}{\beta_{n}^{\prime}}, \quad n \geqslant 1 .
$$

Здесь мы воспользовались тем, что

$$
g(t) \equiv \frac{\alpha(t)}{\beta(t)}=\frac{\alpha_{n}}{\beta_{n}^{\prime}}, \quad t_{n} \leqslant t<t_{n+1} .
$$

С другой стороны,

$$
\mu^{*}(\sigma) \geqslant\left|a_{j_{n}} b_{j_{n}}\right| e^{\lambda_{j_{n}} \sigma}, \quad \lambda_{j_{n}}=t_{n} .
$$

Но $b_{j_{n}}=e^{\alpha\left(t_{n}\right)}=e^{\alpha_{n}}$. Следовательно, для $x_{n-1} \leqslant \sigma<x_{n}$ получаем, что

$$
\ln \mu^{*}(\sigma) \geqslant \alpha_{n}-t_{n} I\left(t_{n}\right)+t_{n} \sigma=\alpha_{n}+\ln \mu(\sigma)>\alpha_{n}, \quad n \geqslant 1 .
$$

Таким образом, для $x_{n-1} \leqslant \sigma<x_{n}$

$$
\frac{\ln \mu(\sigma)}{\ln \mu^{*}(\sigma)} \leqslant \frac{1}{\beta_{n}^{\prime}}
$$


Поскольку $\beta_{n}^{\prime} \rightarrow \infty$ при $n \rightarrow \infty$, то

$$
\lim _{\sigma \rightarrow \infty} \frac{\ln \mu(\sigma)}{\ln \mu^{*}(\sigma)}=0 .
$$

В случае, когда последовательность $\left\{-\ln \left|b_{n}\right|\right\}_{n=N}^{\infty}$ не имеет мажорантной последовательности $\left\{\varphi\left(\lambda_{n}\right)\right\}, \varphi \in W$, пример строится аналогично. Различие состоит только в выборе функций $F(s)$ и $F^{*}(s)$. Действительно, пусть $\alpha(t)$ (мы сохраним для удобства все основные обозначения) - наименышая неубывающая мажоранта для последовательности $\left\{-\ln \left|b_{n}\right|\right\}_{n=N}^{\infty}$. Рассмотрим ряд

$$
F(s)=\sum_{k=1}^{\infty} a_{k}^{\prime} e^{\lambda_{n} s},
$$

где $a_{1}^{\prime}=a_{2}^{\prime}=\cdots=a_{N-1}^{\prime}=1, a_{k}^{\prime}=a_{k} b_{k}^{-1}, k \geqslant N$, причем коэффициенты $a_{k}$ определим по тем же формулам (32). В силу условий (14), (32) этот ряд абсолютно сходится во всей плоскости, т.е. $F \in D(\Lambda)$. Тогда

$$
F^{*}(s)=\sum_{k=1}^{\infty} a_{k}^{\prime} b_{k} e^{\lambda_{n} s}=\sum_{k=1}^{\infty} a_{k}^{\prime \prime} e^{\lambda_{n} s}
$$

где

$$
a_{k}^{\prime \prime}=\left\{\begin{array}{l}
b_{k}, \text { если } 1 \leqslant k \leqslant N-1, \\
a_{k}, \quad \text { если } k \geqslant N .
\end{array}\right.
$$

Ясно, что $F^{*} \in D(\Lambda)$. Те же рассуждения показывают, что в этом случае

$$
\lim _{\sigma \rightarrow \infty} \frac{\ln \mu^{*}(\sigma)}{\ln \mu(\sigma)}=0 .
$$

Необходимость установлена. Тем самым теорема полностью доказана.

\section{§4. Доказательство теоремы 2}

Ясно, что $d(F) \leqslant 1$. Установим оценку снизу.

Пусть $w(x)=\ln M(e x ; Q), w^{*}(x)=\beta(x) w(x), 0<\beta(x) \uparrow \infty,-$ некоторая функция из $W$. В силу условия (7) эта функция существует.

Пусть $v=v(\sigma)$ - решение уравнения $w^{*}(v)=3 \ln \mu(\sigma)$,

$$
h=h(\sigma)=\frac{w^{*}(v(\sigma))}{v(\sigma)} .
$$

Тогда из леммы 1 получаем, что при $\sigma \rightarrow \infty$ вне некоторого множества $E_{\beta}^{\prime} \subset[0, \infty)$ конечной меры

$$
\mu\left(\sigma+\beta^{-1} h(\sigma)\right)=\mu(\sigma)^{1+o(1)}, \quad 0<\beta \leqslant \frac{1}{5} .
$$

Следовательно, при $\sigma \rightarrow \infty$ вне $E_{\beta}^{\prime}$

$$
R_{v}=\sum_{\lambda_{j}>v}\left|a_{j}\right| e^{\lambda_{j} \sigma} \leqslant \mu(\sigma+h(\sigma)) \sum_{\lambda_{j}>v} e^{-h(\sigma) \lambda_{j}}<\mu(\sigma)^{-2(1+o(1))} .
$$


Пусть

$$
P_{a}(s)=\sum_{\lambda_{n} \leqslant a} a_{n} e^{\lambda_{n} s}, \quad s=\sigma+i t
$$

Тогда [21]

$$
a_{n}=e^{-\alpha \lambda_{n}} \frac{1}{2 \pi i} \int_{C} \varphi_{n}(t) P_{a}(t+\alpha) d t
$$

где

$$
\varphi_{n}(t)=\frac{1}{q_{a}^{\prime}\left(\lambda_{n}\right)} \int_{0}^{\infty} \frac{q_{a}(\lambda)}{\lambda-\lambda_{n}} e^{-\lambda t} d \lambda, \quad q_{a}(\lambda)=\prod_{\lambda_{n} \leqslant a}\left(1-\frac{\lambda^{2}}{\lambda_{n}^{2}}\right),
$$

a $C$ - любой замкнутый контур, охватывающий начало координат.

Положим $a=v(\sigma)$, а в качестве $C$ возьмем контур $\{t:|t|=h(\sigma)\}$. Пусть $\alpha=\sigma+i \tau$, где $\tau$ такое, что $\alpha \in \gamma$. Поскольку для $\lambda_{n} \leqslant v(\sigma)$

$$
\frac{1}{\left|q_{v}^{\prime}\left(\lambda_{n}\right)\right|} \leqslant \frac{1}{\left|Q^{\prime}\left(\lambda_{n}\right)\right|}, \quad n \geqslant 1,
$$

то из (35), (36) получаем, что для любого $\lambda_{n} \leqslant v(\sigma)$

$$
\begin{aligned}
\left|a_{n}\right|\left|Q^{\prime}\left(\lambda_{n}\right)\right| e^{\lambda_{n} \sigma} \leqslant & h(\sigma)\left[\max _{|\xi-\alpha| \leqslant h(\sigma)}|F(\xi)|+\sum_{\lambda_{j}>v}\left|a_{j}\right| e^{\lambda_{j}(\sigma+h(\sigma))}\right] \\
& \times \int_{0}^{\infty} M\left(r+2 ; q_{v}\right) e^{-h(\sigma) r} d r .
\end{aligned}
$$

Далее, учитывая определения величин $v=v(\sigma), h=h(\sigma)$, при $\sigma \rightarrow \infty$ имеем

$$
\begin{aligned}
\ln M\left(R ; q_{v}\right) & =n(v) \ln \left(1+\frac{R^{2}}{v^{2}}\right)+2 R^{2} \int_{0}^{v} \frac{n(t)}{t\left(t^{2}+R^{2}\right)} d t \\
& \leqslant \frac{n(v)}{v} R+2 N(v)=o(1) h(\sigma) R+o(1) \ln \mu(\sigma) .
\end{aligned}
$$

Следовательно, учитывая (33) и оценку типа (34), из (37) получаем, что для всех $\lambda_{n} \leqslant v(\sigma)$ при $\sigma \rightarrow \infty$ вне $E_{\beta}^{\prime}$

$$
\left|a_{n}\right|\left|Q^{\prime}\left(\lambda_{n}\right)\right| e^{\lambda_{n} \sigma} \leqslant \mu(\sigma)^{-2(1+o(1))}+\mu(\sigma)^{o(1)} \max _{|\xi-\alpha| \leqslant h(\sigma)}|F(\xi)| .
$$

Пусть $\mu^{*}(\sigma)$ - максимальный член ряда

$$
F^{*}(s)=\sum_{n=1}^{\infty} a_{n} Q^{\prime}\left(\lambda_{n}\right) e^{\lambda_{n} s}
$$

(последовательность $\left\{Q^{\prime}\left(\lambda_{n}\right)\right\}$ удовлетворяет условию (14)). Через $x=x(\sigma)$ обозначим решение уравнения $w^{*}(x)=2 \ln \mu^{*}(\sigma)$. Тогда из леммы 1 получаем, что при $\sigma \rightarrow \infty$ вне некоторого множества $E_{1} \subset[0, \infty)$ конечной меры

$$
R_{x}^{*}=\sum_{\lambda_{j}>x}\left|a_{j}\right|\left|Q^{\prime}\left(\lambda_{j}\right)\right| e^{\lambda_{j} \sigma} \leqslant\left[\mu^{*}(\sigma)\right]^{-1(1+o(1))} .
$$


Следовательно, из $(34),(39)$ при $\sigma \geqslant \sigma_{0}, \sigma \notin E_{1} \cup E_{\beta}^{\prime}$, получаем, что

$$
\lambda_{\mu(\sigma)} \leqslant v(\sigma), \quad \lambda_{k(\sigma)} \leqslant x(\sigma),
$$

где $\nu(\sigma), k(\sigma)$ - центральные индексы рядов (12) и (17).

Далее,

$$
\left|Q^{\prime}\left(\lambda_{n}\right)\right| \leqslant \frac{2}{\lambda_{n}} \prod_{j=1}^{\infty}\left(1+\frac{\lambda_{n}^{2}}{\lambda_{j}^{2}}\right)<M\left(\lambda_{n} ; Q\right), \quad n \geqslant n_{0} .
$$

Следовательно,

$$
\mu^{*}(\sigma)=\left|a_{k} Q^{\prime}\left(\lambda_{k}\right)\right| e^{\lambda_{k} \sigma} \leqslant \mu(\sigma) e^{w(x)},
$$

где $x=x(\sigma), k=k(\sigma)$. Значит, $(1+o(1)) \ln \mu^{*}(\sigma) \leqslant \ln \mu(\sigma)$ при $\sigma \rightarrow \infty$. Так что $w^{*}(x)=2 \ln \mu^{*}(\sigma)<3 \ln \mu(\sigma)=w^{*}(v), \sigma \geqslant \sigma_{1}$. Это означает, что $\lambda_{k(\sigma)} \leqslant x(\sigma)<v(\sigma)$ при $\sigma \geqslant \sigma_{1}, \sigma \notin E_{1} \cup E_{\beta}^{\prime}$. С учетом этого из (38) получаем, что при $\sigma \rightarrow \infty$ вне $E_{1} \cup E_{\beta}^{\prime}$

$$
\mu^{*}(\sigma)<1+\mu(\sigma)^{o(1)} \max _{|\xi-\alpha| \leqslant h(\sigma)}|F(\xi)|
$$

где $\alpha=\sigma+i \tau, \alpha \in \gamma$.

Дальнейшие рассуждения основаны на лемме 3 .

Пусть $\gamma(\alpha)$ - часть кривой, содержащейся в круге $\bar{D}\left(\alpha ; h \beta^{-1}\right), 0<\beta \leqslant 1 / 5$, $h=h(\sigma)$. Она не обязательно кусочно-гладкая и жорданова. Поэтому поступаем следующим образом.

Пусть $\alpha_{1}$ - какая-нибудь точка кривой $\gamma(\alpha)$, принадлежашая окружности $\partial \bar{D}\left(\alpha ; h \beta^{-1}\right)$. Соединим точку $\alpha_{1}$ с $\alpha$ ломаной $l(\alpha)$ без самопересечений и такой, что

$$
\rho(\xi)=\inf _{z \in \gamma(\alpha)}|F(z)-F(\xi)| \leqslant m_{\alpha}
$$

где $\xi \in l(\alpha), m_{\alpha}=\max _{\gamma(\alpha)}|F(t)|$. Это можно сделать. Действительно, для любого $z \in \gamma(\alpha)$ существует окрестность $D\left(z ; \nu_{z}\right), \nu_{z}>0$, такая, что

$$
|F(z)-F(\xi)| \leqslant m_{\alpha}
$$

для любого $\xi \in D\left(z ; \nu_{z}\right)$. Поскольку $\gamma(\alpha)$ - компакт, то по лемме Гейне-Бореля из покрытия $\bigcup_{z \in \gamma(\alpha)} D\left(z ; \nu_{z}\right)$ можно выделить конечное подпокрытие: $D\left(z_{0} ; \nu_{0}\right)$, $D\left(z_{1} ; \nu_{1}\right), \ldots, D\left(z_{n} ; \nu_{n}\right)$, где $z_{i} \in \gamma(\alpha), \nu_{i}>0, i=0,1, \ldots, n$.

Можем считать, что $z_{0}=\alpha, z_{n}=\alpha_{1}$. Соединим между собой центры всех попарно пересекающихся кружков $D\left(z_{i} ; \nu_{i}\right), i=0,1, \ldots, n$, отрезками прямых. Отбрасывая, если это необходимо, конечное число отрезков, получим жорданову ломаную $l(\alpha)$, соединяющую точки $\alpha$ и $\alpha_{1}$. Заметим, что $l(\alpha)$ целиком содержится в объединении кружков $D\left(z_{i} ; \nu_{i}\right), i=0,1, \ldots, n$. Осталось проверить оценку $(41)$.

Пусть $\xi \in l(\alpha)$. Тогда сушествует круг $D\left(z_{i} ; \nu_{i}\right)$, содержащий эту точку. Поскольку $\left|F\left(z_{i}\right)-F(\xi)\right| \leqslant m_{\alpha}$, то $\rho(\xi) \leqslant m_{\alpha}$.

Вернемся теперь к оценке (40). Применяя лемму 3 для круга $\bar{D}\left(z_{0} ; \beta^{-1} h\right)$ и кривой $l(\alpha)$, получаем

$$
\begin{aligned}
\max _{|\xi-\alpha| \leqslant h(\sigma)}|F(\xi)| & \leqslant \max _{l(\alpha)}|F(t)|^{1-2 \beta} \max _{|t-\alpha| \leqslant \beta^{-1} h(\sigma)}|F(t)|^{2 \beta} \\
& \leqslant\left|F\left(\xi_{\alpha}\right)\right|^{1-2 \beta} M^{2 \beta}\left(\sigma+\beta^{-1} h(\sigma)\right), \quad \xi_{\alpha} \in l(\alpha) .
\end{aligned}
$$


Но из (41) следует, что

$$
\rho\left(\xi_{\alpha}\right)=\left|F\left(\xi_{\alpha}\right)-F\left(z_{\alpha}\right)\right| \leqslant m_{\alpha}
$$

для некоторой точки $\left.z_{\alpha} \in \gamma_{(} \alpha\right)$. Следовательно,

$$
\begin{aligned}
\max _{|\xi-\alpha| \leqslant h(\sigma)}|F(\xi)| & \leqslant\left[2 m_{\alpha}\right]^{1-2 \beta} M^{2 \beta}\left(\sigma+\beta^{-1} h(\sigma)\right) \\
& =\left(2\left|F\left(z_{\alpha}^{\prime}\right)\right|\right)^{1-2 \beta} M^{2 \beta}\left(\sigma+\beta^{-1} h(\sigma)\right),
\end{aligned}
$$

где $z_{\alpha}^{\prime} \in \gamma_{\alpha}$.

Применяя лемму 1 , имеем

$$
\begin{aligned}
\mu(\sigma) & \leqslant M(\sigma)=M\left(\sigma+\beta^{-1} h(\sigma)\right) \leqslant \sum_{n=1}^{\infty}\left|a_{n}\right| e^{\lambda_{n}\left(\sigma+\beta^{-1} h(\sigma)\right)} \\
& \leqslant \mu\left(\sigma+\left(1+\beta^{-1}\right) h(\sigma)\right)\left[n(v(\sigma))+\sum_{\lambda_{n}>v(\sigma)} e^{-h(\sigma) \lambda_{n}}\right]<\mu(\sigma)^{1+o(1)}
\end{aligned}
$$

при $\sigma \rightarrow \infty$ вне некоторого множества $E_{\beta}^{\prime \prime} \subset[0, \infty)$ конечной меры. Следовательно, с учетом оценок (42), (43) из (40) получаем, что при $\sigma \rightarrow \infty$ вне множества $E_{\beta}=$ $E_{1} \cup E_{\beta}^{\prime} \cup E_{\beta}^{\prime \prime}$ конечной меры

$$
(1+o(1)) \mu^{*}(\sigma) \leqslant 2\left|F\left(z_{\alpha}^{\prime}\right)\right|^{1-2 \beta} \mu(\sigma)^{(1+o(1)) 2 \beta}, \quad z_{\alpha}^{\prime} \in \gamma(\alpha) .
$$

Следовательно, при $\sigma \rightarrow \infty$ вне $E_{\beta}$

$$
\frac{\ln \mu^{*}(\sigma)}{\ln \mu(\sigma)} \leqslant(1+o(1)) 2 \beta+(1-2 \beta) \frac{\ln \left|F\left(z_{\alpha}^{\prime}\right)\right|}{\ln \mu(\sigma)}, \quad 0<\beta \leqslant \frac{1}{5}
$$

Учитывая еще раз оценки (43), отсюда получаем, что

$$
\varlimsup_{\substack{\sigma \in e_{\beta} \\ \sigma \rightarrow \infty}} \frac{\ln \mu^{*}(\sigma)}{\ln \mu(\sigma)} \leqslant 2 \beta+(1-2 \beta) d(F ; \gamma)
$$

где $0<\beta \leqslant 1 / 5, e_{\beta}=[0, \infty) \backslash E_{\beta}$. Следовательно, из (44) имеем

$$
q(F)=\inf _{e} \varlimsup_{\substack{\sigma \in e \\ \sigma \rightarrow \infty}} \frac{\ln \mu^{*}(\sigma)}{\ln \mu(\sigma)} \leqslant 2 \beta+(1-2 \beta) d(F ; \gamma),
$$

где величина $q(F)$ вычисляется по всем множествам $e \subset \mathbb{R}_{+}$, каждое из которых получается исключением из $\mathbb{R}_{+}$некоторой системы отрезков конечной суммарной длины. Поскольку левая часть этого неравенства от $\beta$ и $\gamma$ не зависит, то, сначала устремляя $\beta$ к нулю, а затем минимизируя правую часть по всем $\gamma \in \Gamma$, получаем требуемое неравенство (19).

Теорема полностью доказана.

Из теоремы 2 следует, что $0 \leqslant q(F) \leqslant 1$. Можно показать, что в условиях теоремы А справедливы равенства $q(F)=d(F)=1$ (см. $\S 5$ ). Возникает вопрос: возможна ли оценка $q(F)>0$ при выполнении единственного условия (7) (при этом условие (9) вообще может не вьполняться)?

Имеет место следующее

УТВЕРЖДЕНИЕ. Для любой последовательности $\Lambda$ с лакунами Фейера, для которой интеграл (9) расходится, существует функиия $F \in D(\Lambda)$ такая, чmо $q(F)=d(F)=1$. 
ДокАЗАТЕЛЬСтво. Пусть условие (9) не вьполнено, т.е.

$$
\int_{1}^{\infty} \frac{\alpha(t)}{t^{2}} d t=\infty
$$

Напомним, что $\alpha(t)=0$ при $0 \leqslant t<\lambda_{1}, \alpha(t)=\max _{\lambda_{n} \leqslant t}\left\{\ln ^{+} \frac{1}{\left|Q^{\prime}\left(\lambda_{n}\right)\right|}\right\}$ при $\lambda_{1} \leqslant t$. Функция $\alpha(t)$ неотрицательная, неубывающая, непрерывная справа. Пусть $\left\{t_{n}\right\}$, $0<t_{n} \uparrow \infty, t_{n}=\lambda_{k_{n}},-$ последовательность всех точек разрыва $\alpha(t)$, причем $\alpha(t)=\alpha_{n}$ при $t_{n} \leqslant t<t_{n+1}, n \geqslant 1$. Сушествует подпоследовательность $\left\{t_{n_{m}}\right\}$ такая, что ступенчатая функция $b(t)=b_{m}$ при $\mu_{m} \leqslant t<\mu_{m+1}, \mu_{m}=t_{n_{m}}$, $b_{m}=\alpha\left(\mu_{m}\right)$, обладает свойствами:

$$
0 \leqslant b(t) \leqslant \alpha(t), \quad \int_{\mu_{1}}^{\infty} \frac{b(t)}{t^{2}} d t<\infty .
$$

Можно считать, что $\mu_{m} \geqslant 1, m \geqslant 1$.

Пусть $y=\gamma_{1}(x), y=\gamma_{2}(x), x \geqslant \mu_{1},-$ уравнения выпуклых полигонов Ньютона для рядов

$$
g(s)=\sum_{m=1}^{\infty} c_{m} e^{\mu_{m} s}, \quad g^{*}(s)=\sum_{m=1}^{\infty} c_{m} Q^{\prime}\left(\mu_{m}\right) e^{\mu_{m} s},
$$

где $c_{m}=e^{-\mu_{m} \ln \mu_{m}}$. Ясно, что ряды сходятся во всей плоскости. Рассмотрим теперь ряды Дирихле

$$
F(s)=\sum_{m=1}^{\infty} a_{k} e^{\lambda_{k} s}, \quad F^{*}(s)=\sum_{m=1}^{\infty} a_{k} Q^{\prime}\left(\lambda_{k}\right) e^{\lambda_{k} s},
$$

где

$$
a_{k}= \begin{cases}c_{m}, & \text { если } \lambda_{k}=\mu_{m}, \\ e^{-\gamma\left(\lambda_{k}\right)-\beta\left(\lambda_{k}\right)-1}, & \text { если } \lambda_{k} \neq \mu_{m} .\end{cases}
$$

Здесь $\gamma(x)=\max \left(\gamma_{1}(x), \gamma_{2}(x)\right)$, а $\beta\left(\lambda_{k}\right)=\ln ^{+}\left|Q^{\prime}\left(\lambda_{k}\right)\right|$. Поскольку $\gamma(x) / x \rightarrow \infty$ при $x \rightarrow \infty$, то видно, что $F \in D(\Lambda), F^{*} \in D(\Lambda)$, а так как при $\lambda_{k} \neq \mu_{m}$ имеют место оценки $\left|a_{k}\right| \leqslant e^{-\gamma_{1}\left(\lambda_{k}\right)-1},\left|a_{k} Q^{\prime}\left(\lambda_{k}\right)\right| \leqslant e^{-\gamma_{2}\left(\lambda_{k}\right)-1}$, то максимальные члены последних рядов совпадают с максимальными членами первых рядов соответственно [20; гл. $2, \S 6$, п. 2]:

$$
\mu(\sigma)=\max _{m \geqslant 1}\left\{\left|c_{m}\right| e^{\mu_{m} \sigma}\right\}, \quad \mu^{*}(\sigma)=\max _{m \geqslant 1}\left\{\left|c_{m} B_{m}\right| e^{\mu_{m} \sigma}\right\},
$$

где $B_{m}=Q^{\prime}\left(\mu_{m}\right)=e^{b_{m}}, m \geqslant 1$. Поскольку $b_{m} \geqslant 0$, то

$$
\left|B_{m}\right|+\frac{1}{\left|B_{m}\right|} \leqslant e^{b_{m}}+1 \leqslant e^{\ln 2+b\left(\mu_{m}\right)}, \quad m \geqslant 1 .
$$

Но $\ln 2+b\left(\mu_{m}\right) \leqslant w\left(\mu_{m}\right), m \geqslant 1$, где $w$ - некоторая функция из $W$. Следовательно, из теоремы 1 получаем, что при $\sigma \rightarrow \infty$ вне некоторого множества $E \subset \mathbb{R}_{+}$ конечной меры

$$
\ln \mu^{*}(\sigma)=(1+o(1)) \ln \mu(\sigma) .
$$

Следовательно, $q(F)=d(F)=1$ (см. следствие 1$)$.

Утверждение доказано. 


\section{§5. Доказательство теоремы 3}

1. Достаточность доказана в [23]. Приведем другое доказательство, основанное на теоремах 1,2 .

Из условий (7), (9) следует, что

$$
\left|Q^{\prime}\left(\lambda_{n}\right)\right|+\frac{1}{\left|Q^{\prime}\left(\lambda_{n}\right)\right|} \leqslant e^{w\left(\lambda_{n}\right)}, \quad n \geqslant 1,
$$

для некоторой функции $w \in W$. Следовательно, применяя теорему 1 , из (19) получаем, что

$$
q(F)=d(F)=1 .
$$

2. Необходимость. Пусть условие (9) не выполнено, т.е.

$$
\int_{1}^{\infty} \frac{\alpha(t)}{t^{2}} d t=\infty
$$

Ясно, что $\alpha(t)$ - неотрицательная неубывающая ступенчатая функция, непрерывная справа. Пусть $\left\{t_{n}\right\}, 0<t_{n} \uparrow \infty,-$ последовательность всех точек разрьва $\alpha(t)$, и пусть $\alpha(t)=\alpha_{n}$ при $t_{n} \leqslant t<t_{n+1}, n \geqslant 1$. Из условия (7) следует, что [21]

$$
\int_{1}^{\infty} \frac{\ln M(r ; Q)}{r^{2}} d r<\infty
$$

где $M(r ; Q)$ - функция из формулы (18). Следовательно, существует уточненный порядок $\rho(r)$ такой, что [32]

$$
2 \ln \left(1+r^{2}\right)+20 \ln M(r ; Q) \leqslant r^{\rho(r)}, \quad r \geqslant 1, \quad \int_{1}^{\infty} r^{\rho(r)-2} d r<\infty .
$$

Пусть $w=w(r)$ - функция из $W$ такая, что $r^{\rho(r)}=o(w(r))$ при $r \rightarrow \infty$. Из (45), (46) следует, что множество индексов

$$
J=\left\{n: n \geqslant 1, \alpha\left(t_{n}\right)>w\left(t_{n}\right)\right\}
$$

не ограничено. Пусть $I_{j}=\left[\tau_{j}, \tau_{j}^{\prime}\right), \tau_{j}<\tau_{j}^{\prime}, j \geqslant 1,-$ максимальные интервалы, на которых $w(t) \geqslant \alpha(t)$. Ясно, что $I_{i} \cap I_{j}=\varnothing, i \neq j$. Положим $I=\bigcup_{j} I_{j}$,

$$
\alpha^{*}(t)= \begin{cases}\alpha(t), & \text { если } t \notin I, \\ \min _{x \in I_{j}} \alpha(x), & \text { если } t \in I_{j} .\end{cases}
$$

Подправленная таким образом функция $\alpha^{*}(t)$ неубьвающая, непрерывная справа, $0 \leqslant \alpha^{*}(t) \leqslant \alpha(t)$. Поскольку $w \in W$, то

$$
\int_{I} \frac{\alpha(t)}{t^{2}} d t \leqslant \int_{I} \frac{w(t)}{t^{2}} d t<\infty .
$$


Следовательно, с учетом (45) заключаем, что

$$
\int_{1}^{\infty} \frac{\alpha^{*}(t)}{t^{2}} d t=\infty
$$

Пусть $\left\{x_{j}\right\}$ - последовательность всех точек разрыва функции $\alpha^{*}(t)$. Поскольку функция $\alpha(t)$ ступенчатая, а $w(t)$ непрерывная и возрастающая, то $\left\{x_{j}\right\}$ есть подпоследовательность $\left\{t_{n}\right\}$, т.е. $x_{j}=\lambda_{n_{j}}, j \geqslant 1$. Пусть $\alpha^{*}(t)=\alpha_{j}^{*}$ при $x_{j} \leqslant t<x_{j+1}$. Из условия (47) следует (см. $\S 3$ ) существование неубывающей функции $g(t)=\alpha^{*}(t) \beta^{-1}(t)\left(\beta(t)\right.$ - неограниченная неубывающая на $\left[\lambda_{1}, \infty\right)$ функция, $\beta(t)>0, \beta(t) \equiv$ const $=\beta_{j}$ при $\left.x_{j} \leqslant t<x_{j+1}, j \geqslant 1\right)$ такой, что

$$
\begin{gathered}
\int_{\lambda_{1}}^{\infty} \frac{g(t)}{t^{2}} d t=\infty, \\
g(t)= \begin{cases}g_{j}, & \text { если } x_{j} \leqslant t<x_{j+1}, j \geqslant 1, \\
g_{1}, & \text { если } \lambda_{1} \leqslant t<x_{1},\end{cases}
\end{gathered}
$$

где $g_{j}=\alpha_{j}^{*} / \beta_{j}, j \geqslant 1,0<\beta_{j} \rightarrow \infty, j \rightarrow \infty$.

Далее, из леммы 2 и следствия 3 вытекает сушествование окружностей

$$
K_{n}=\left\{z:|z|=r_{n}\right\}, \quad \lambda_{1}<r_{n} \uparrow \infty, \quad r_{n-1}<r_{n} \leqslant 4 r_{n}
$$

таких, что

$$
\frac{1}{|Q(z)|} \leqslant M^{20}(r ; Q), \quad|z|=r=r_{n}, \quad n \geqslant 1
$$

Пусть $X=\left\{x_{j}\right\}$, а $\Delta_{j}=\left(r_{j}^{\prime}, r_{j}^{\prime \prime}\right), r_{j}^{\prime}=r_{n_{j}}, r_{j}^{\prime \prime}=r_{n_{j}+1},-$ все интервалы, каждьй из которых содержит хотя бы одну точку из $X$. Здесь $r_{n_{j}}$ - подпоследовательность последовательности $r_{n}$, фигурирующей в оценках (49). Пусть, далее, $G_{j}=d_{j} h_{j}$, где

$$
d_{j}=\min _{k}\left\{x_{k}: x_{k} \in \Delta_{j} \cap \Lambda\right\}, \quad h_{j}=\int_{\lambda_{1}}^{d_{j}} \frac{g(t)}{t^{2}} d t .
$$

Из условия (48) следует, что $h_{j} \uparrow \infty$ при $j \rightarrow \infty$. В $\S 3$ было показано, что в этом случае

$$
R_{j}=\frac{G_{j+1}-G_{j}}{d_{j+1}-d_{j}} \uparrow \infty .
$$

Соединяя попарно все точки $P_{j}$ и $P_{j+1}, j \geqslant 1$, отрезками прямых, получаем некоторый выпуклый полигон (ломаную) $L$. Если $y=\varphi(t)-$ уравнение $L$, то ясно, что

$$
\lim _{t \rightarrow \infty} \frac{\varphi(t)}{t}=+\infty
$$

Пусть $\rho(r)$ - уточненный порядок, введенный вьше, а $\left\{\nu_{n}\right\}$ - последовательность положительных чисел такая, что

$$
\frac{n}{\nu^{\rho\left(\nu_{n}\right)}}=1, \quad n \geqslant 1
$$


Положим

$$
P(z)=\prod_{n=1}^{\infty}\left(1+\frac{z^{2}}{\nu_{n}^{2}}\right)
$$

Если $z=r e^{i \varphi},|\varphi| \leqslant \pi / 4$, то согласно лемме 4 сушествует равномерный по $\varphi$ предел

$$
\lim _{r \rightarrow \infty} \frac{\ln \left|P\left(r e^{i \varphi}\right)\right|}{r^{\rho(r)}}=\pi \cos \varphi .
$$

Рассмотрим теперь ряд Дирихле

$$
F(s)=\sum_{k=1}^{\infty} a_{k} e^{\lambda_{k} s}, \quad s=\sigma+i t,
$$

коэффициенты которого определим следуюшим образом:

$$
a_{k}= \begin{cases}\exp \left(-\lambda_{k} h_{j}\right) \frac{1}{Q^{\prime}\left(\lambda_{k}\right) P\left(\lambda_{k}\right)}, & \text { если } \lambda_{k} \in \Delta_{j}, j \geqslant 1, \\ \frac{1}{\lambda_{k}} \exp \left(-\varphi_{j}\left(\lambda_{k}\right)-1\right), & \text { если } \lambda_{k} \in \Delta_{j}^{\prime}, j \geqslant 1, \\ 1, & \text { если } \lambda_{1} \leqslant \lambda_{k}<r_{1}^{\prime},\end{cases}
$$

где $\Delta_{j}=\left(r_{j}^{\prime}, r_{j}^{\prime \prime}\right), \Delta_{j}^{\prime}=\left(r_{j}^{\prime \prime}, r_{j+1}^{\prime}\right), y_{j}=\varphi_{j}(t)$ - уравнение прямой, проходяшей через точки $P_{j}$ и $P_{j+1}$.

По предположению последовательность $\Lambda$ имеет конечный индекс конденсации, а $P\left(\lambda_{k}\right) \geqslant 1, k \geqslant 1$. Поскольку $h_{i} \uparrow \infty$ и вьполняется условие (52), то ряд Дирихле (54) абсолютно сходится во всей плоскости, т.е. $F \in B(\Lambda)$.

Оценим функцию $F(s)$ на вешественной оси сверху.

Пусть $\Gamma_{n}$ - контур, составленный отрезками лучей $\{\mu: \arg \mu= \pm \pi / 4\}$, соединяющими окружности $K_{n}$ и $K_{n+1}$, и дугами этих окружностей. Если

$$
F_{j}(\sigma)=\sum_{\lambda_{k} \in \Delta_{j}} a_{k} e^{\lambda_{k} \sigma}, \quad j \geqslant 1
$$

то имеем

$$
F_{j}(\sigma)=\frac{1}{2 \pi i} \int_{C_{j}} \frac{e^{-\mu h_{j}+\mu \sigma}}{Q(\mu) P(\mu)} d \mu,
$$

где $\Delta_{j}=\left(r_{j}^{\prime}, r_{j}^{\prime \prime}\right), r_{j}^{\prime}=r_{n_{j}}, r_{j}^{\prime \prime}=r_{n_{j}+1}, C_{j}=\Gamma_{n_{j}}$, а величина $h_{j}$ определена в (50).

Оценим теперь функцию $F_{j}(\sigma)$. Для этого сначала оценим функции $Q(\mu)$ и $P(\mu)$ на контуре $C_{j}$.

Поскольку $\left|Q\left(r e^{ \pm \frac{\pi}{4} i}\right)\right| \geqslant 1$, то с учетом (49) и (53) на контуре $C_{j}, j \geqslant 1$, получаем, что для любого $\varepsilon>0$

$$
|\mu|\left(1+|\mu|^{2}\right) \frac{1}{|Q(\mu) P(\mu)|} \leqslant \exp \left[2 \ln \left(1+r^{2}\right)+20 \ln M(r ; Q)-\left(\pi \frac{\sqrt{2}}{2}-\varepsilon\right) r^{\rho(r)}\right],
$$

где $|\mu|=r, r_{j}^{\prime} \leqslant r \leqslant r_{j}^{\prime \prime}, j \geqslant j_{0}$. 
Воспользуемся теперь оценкой из (46). Тогда

$$
\max _{\mu \in C_{j}}\left(3|\mu|\left(1+|\mu|^{2}\right) \frac{1}{|Q(\mu) P(\mu)|}\right) \leqslant A<\infty, \quad j \geqslant 1,
$$

где $A-$ постоянная, не зависящая от $j \geqslant 1$. Если

$$
L_{j}(\mu)=\frac{e^{-\mu h_{j}+\mu \sigma}}{Q(\mu) P(\mu)},
$$

TO

$$
\left|F_{j}(\sigma)\right| \leqslant\left(\frac{1}{2 \pi} \int_{C_{j}}|d \mu|\right) \max _{\mu \in C_{j}}\left|L_{j}(\mu)\right|=\left(\frac{1}{2 \pi} \int_{C_{j}}|d \mu|\right)\left|L_{j}\left(\mu_{j}\right)\right|,
$$

где $\mu_{j}$ - некоторая точка, принадлежащая контуру $C_{j}$.

Далее, поскольку $r_{j}^{\prime}<r_{j}^{\prime \prime}<4 r_{j}^{\prime}$, то

$$
\frac{1}{2 \pi} \int_{C_{j}}|d \mu| \leqslant 3\left|\mu_{j}\right|, \quad j \geqslant 1
$$

Следовательно, учитывая оценку (55), имеем

$$
\begin{aligned}
\left|F_{j}(\sigma)\right| & \leqslant \frac{3\left|\mu_{j}\right|}{1+\left|\mu_{j}\right|^{2}} \frac{1+\left|\mu_{j}\right|^{2}}{\left|Q\left(\mu_{j}\right) P\left(\mu_{j}\right)\right|} \exp \left[-\left(\operatorname{Re} \mu_{j}\right)\left(h_{j}-\sigma\right)\right] \\
& \leqslant \frac{A}{1+\left|\mu_{j}\right|^{2}} \exp \left(-\gamma_{j} h_{j}+\gamma_{j} \sigma\right)
\end{aligned}
$$

где $\gamma_{j}=\operatorname{Re} \mu_{j}, r_{j}^{\prime} \leqslant \gamma_{j} \leqslant r_{j}^{\prime \prime}, j \geqslant 1$.

Таким образом,

$$
|F(\sigma)| \leqslant \sum_{j=1}^{\infty} \frac{A}{1+\left|\mu_{j}\right|^{2}} e^{-\gamma_{j} h_{j}+\gamma_{j} \sigma}+R(\sigma),
$$

где

$$
R(\sigma)=\sum_{\lambda_{k} \notin \Delta}\left|a_{k}\right| e^{\lambda_{k} \sigma}, \quad \Delta=\bigcup_{j=1}^{\infty} \Delta_{j}
$$

Отсюда получаем, что

$$
|F(\sigma)| \leqslant B \exp \left[\max _{j \geqslant 1}\left(-\gamma_{j} h_{j}+\gamma_{j} \sigma\right)\right]+R(\sigma)
$$

Поскольку $h_{j} \uparrow \infty$ при $j \rightarrow \infty$, то $\max _{j \geqslant 1}\left(-\gamma_{j} h_{j}+\gamma_{j} \sigma\right)=H(\sigma)$ сушествует при любом фиксированном $\sigma>0$. Далее, при $\sigma \geqslant \sigma_{0}$ имеем: $0<H(\sigma)=-\gamma_{\nu} h_{\nu}+$ $\gamma_{\nu} \sigma \leqslant 4 r_{\nu}^{\prime}\left(-h_{\nu}+\sigma\right)<4 d_{\nu}\left(-h_{\nu}+\sigma\right)$, где $r_{\nu}^{\prime} \leqslant \gamma_{\nu} \leqslant r_{\nu}^{\prime \prime}, d_{\nu}$ - ближайшая к $r_{\nu}^{\prime}$ точка скачка функции $g(t)$ из интервала $\Delta_{\nu}$. Тогда из (56) получаем, что

$$
|F(\sigma)| \leqslant B \exp \left[4 \max _{j \geqslant 1}\left(-d_{j} h_{j}+d_{j} \sigma\right)\right]+R(\sigma)
$$

где $d_{j}$ - ближайшая к $r_{j}^{\prime}$ точка скачка функции $g(t)$ из интервала $\Delta_{j}=\left(r_{j}^{\prime}, r_{j}^{\prime \prime}\right)$. 
Пусть $R_{n-1} \leqslant \sigma<R_{n}$, где числа $R_{n}$ определены формулой (51). Поскольку $R_{n} \uparrow \infty$, то из $\S 3$ следует, что для таких $\sigma$

$$
\max _{j \geqslant 1}\left(-d_{j} h_{j}+d_{j} \sigma\right)=-d_{n} h_{n}+d_{n} \sigma=m(\sigma)
$$

Коэффициенты ряда $R(\sigma)$ выбраны нами специальньм образом, а именно они таковы, что построенная выше ломаная $L$ является полигоном Ньютона для ряда

$$
\sum_{m=1}^{\infty} b_{m} e^{\mu_{m} \sigma}=\sum_{j=1}^{\infty} e^{-d_{j} h_{j}} e^{d_{j} \sigma}+R(\sigma)
$$

где $\left\{\mu_{m}\right\}=\left\{d_{j}\right\} \cup\left\{\lambda_{k}: \lambda_{k} \in \Lambda \backslash \Delta\right\}$

Следовательно, при $R_{n-1} \leqslant \sigma<R_{n}$ из $(7),(57),(58)$ получаем (см. $\left.\S 3\right)$, что

$$
|F(\sigma)| \leqslant B e^{4 m(\sigma)}+\sum_{k=1}^{\infty} \frac{1}{\lambda_{k}} e^{m(\sigma)} \leqslant C e^{4 m(\sigma)} .
$$

Оценим $m(\sigma)$ сверху. Для $R_{n-1} \leqslant \sigma<R_{n}$ имеем

$$
m(\sigma)=-d_{n} h_{n}+d_{n} \sigma \leqslant \frac{d_{n} d_{n+1}}{d_{n+1}-d_{n}} \int_{d_{n}}^{d_{n+1}} \frac{g(t)}{t^{2}} d t \leqslant g\left(t_{n}^{\prime}\right),
$$

где $t_{n}^{\prime}$ - наибольшая точка скачка функции $g(t)$ из интервала $\left(r_{n}^{\prime}, r_{n}^{\prime \prime}\right)$ (на участке $\left[t_{n}^{\prime}, d_{n+1}\right)$ функция $g(t)$ постоянна!). Поскольку по построению

$$
g(t) \equiv \frac{\alpha^{*}\left(t_{n}^{\prime}\right)}{\beta\left(t_{n}^{\prime}\right)}, \quad t_{n}^{\prime} \leqslant t<d_{n+1},
$$

то при $R_{n-1} \leqslant \sigma<R_{n}$

$$
m(\sigma) \leqslant g\left(t_{n}^{\prime}\right)=\frac{\alpha^{*}\left(t_{n}^{\prime}\right)}{\beta\left(t_{n}^{\prime}\right)} \leqslant \frac{\alpha\left(t_{n}^{\prime}\right)}{\beta\left(t_{n}^{\prime}\right)} .
$$

С другой стороны, для тех же $\sigma$ имеем:

$$
\ln \mu(\sigma) \geqslant \ln \left|a_{n}^{\prime}\right|+t_{n}^{\prime} \sigma
$$

где $a_{n}^{\prime}$ - коэффициент ряда (54), соответствующий показателю $t_{n}^{\prime}$, а $\mu(\sigma)$ - максимальный член этого ряда. Поскольку $r_{n}^{\prime}<t_{n}^{\prime}<r_{n}^{\prime \prime}$, то с учетом (58), (53) для $R_{n-1} \leqslant \sigma<R_{n}$ получаем, что

$$
\begin{aligned}
\ln \mu(\sigma) & \geqslant-t_{n}^{\prime} h_{n}+t_{n}^{\prime} \sigma+\ln \frac{1}{\left|Q^{\prime}\left(t_{n}^{\prime}\right)\right|}+\ln \frac{1}{\left|P\left(t_{n}^{\prime}\right)\right|} \\
& \geqslant \frac{r_{n}^{\prime}}{r_{n}^{\prime \prime}} m(\sigma)+\alpha\left(t_{n}^{\prime}\right)+\ln \frac{1}{\left|P\left(t_{n}^{\prime}\right)\right|}>\alpha\left(t_{n}^{\prime}\right)-2 \pi\left(t_{n}^{\prime}\right)^{\rho\left(t_{n}^{\prime}\right)} \\
& =\alpha\left(t_{n}^{\prime}\right)+o(1) w\left(t_{n}^{\prime}\right)=(1+o(1)) \alpha\left(t_{n}^{\prime}\right), \quad n \rightarrow \infty
\end{aligned}
$$

Здесь мы учли то, что $t_{n}^{\prime} \in X$, т.е. $w\left(t_{n}^{\prime}\right) \leqslant \alpha\left(t_{n}^{\prime}\right)$. 
Таким образом, для $R_{n-1} \leqslant \sigma<R_{n}$ из (59)-(61) окончательно получаем, что при $n \rightarrow \infty$

$$
\frac{\ln |F(\sigma)|}{\ln \mu(\sigma)} \leqslant \frac{\ln C}{\ln \mu(\sigma)}+4 \frac{m(\sigma)}{\ln \mu(\sigma)} \leqslant o(1)+(1+o(1)) \frac{1}{\beta\left(t_{n}^{\prime}\right)} .
$$

Далее, из (61) видно, что $\ln \mu(\sigma) \rightarrow \infty$ при $\sigma \rightarrow \infty$, а поскольку

$$
\sum_{n=1}^{\infty} \frac{1}{\lambda_{n}}<\infty
$$

то из результатов работы [27] вытекает, что $\sup _{\sigma>0}|F(\sigma)|=\infty$.

Следовательно, из (62) окончательно получаем, что

$$
d\left(F ; \mathbb{R}_{+}\right)=\varlimsup_{\sigma \rightarrow \infty} \frac{\ln |F(\sigma)|}{\ln M(\sigma)}=0 .
$$

Теорема полностью доказана.

В заключение автор благодарит В. В. Напалкова, И. Ф. Красичкова-Терновского и других участников семинара по теории функций комплексного переменного за обсуждение работы.

\section{Список литературы}

1. Fejér L. Über die Wurzel vom kleinsten absoluten Betrage einer algebraischen Gleichung // Math. Ann. 1908. P. 413-423.

2. Macintyre A.J. Asymptotic paths of integral functions with gap power series // Proc. London Math. Soc. (3). 1952. V. 2. № 2. P. 286-296.

3. Pólya G. Untersuchungen über Lücken und Singularitäten von Potenzeihen // Math. Z. 1929. V. 29. P. 549-640.

4. Fuchs W. H. J. Proof of a conjecture of G. Pólya concerning gap series // Illinois J. Math. 1963. V. 7. P. 661-667.

5. Kövari T. A gap-theorem for entire functions of infinite order // Michigan Math. J. 1965 . V. 12. № 2. P. 133-140.

6. Kövari T. On the asymptotic paths of entire functions with gap power series // J. Anal. Math. 1965. V. 15. P. 281-286.

7. Sons L. R. On the Macintyre conjecture // Illinois J. Math. 1970. V. 14. P. 613-629.

8. Sons L. R. An analogue of a theorem of W. H. J. Fuchs on gap series // Proc. London Math. Soc. (3). 1970. V. 21. №3. P. 525-539.

9. Hayman W. K. Angular value distribution of power series with gaps // Proc. London Math. Soc. (3). 1972. V. 24. P. 590-624.

10. Павлов А.И. О росте по кривым целых функций, заданных лакунарными степенными рядами // Сиб. матем. журн. 1972. Т. 13. №5. С. 1169-1181.

11. Павлов А.И. О росте на положительном луче целых функций с вещественньми коэффициентами Тейлора // Матем. заметки. 1973. Т. 14. № 4. С. 577-588.

12. Korevaar J., Dixon $M$. Interpolation, strongly nonspanning powers and Macintyre exponents // Indag. Math. (N.S.). 1978. V. 40. № 2. P. 243-258.

13. Berndtsson B. A note on Pavlov-Korevaar-Dixon interpolation // Indag. Math. (N.S.). 1978. V. 81. P. 400-414.

14. Murai T. The deficiency of entire functions with Fejer gaps // Ann. Inst. Fourier (Grenoble). 1983. V. 33. №3. P. 39-58. 
15. Гайсин A. M. Асимптотическое поведение суммы целого ряда Дирихле на кривых // Исследования по теории приближений. Уфа: БНЦ УрО АН СССР, 1989. С. 3-15.

16. Гайсин A. М. Усиленная неполнота системы экспонент и проблема Макинтайра // Матем. сб. 1991. Т. 182. № 7. С. 931-945.

17. Skaskiv O.B. On the Pólya conjecture concerning the maximum and minimum of the modulus of an entire function of finite order given by a lacunary power series // Anal. Math. 1990. V. 16. № 2. P. 143-157.

18. Гайсин А. М. Об одной гипотезе Полиа // Изв. РАН. Сер. матем. 1994. Т. 58. № 2. C. 73-92.

19. Гайсин A. М. Асимптотические свойства функций, заданных рядами экспонент // Дис. ... докт. физ.-матем. наук. Уфо, 1994.

20. Леонтьев А. Ф. Ряды экспонент. М.: Наука, 1976.

21. Леонтьев А. Ф. Последовательности полиномов из экспонент. М.: Наука, 1980.

22. Гайсин A. M. Об одной теореме Хеймана // Сиб. матем. журн. 1998. Т. 39. № 3. C. 501-516.

23. Гайсин A.M. Асимптотическая оценка суммы ряда Дирихле на кривых // Матем. заметки. 1997. Т. 61. №6. С. 810-816.

24. Шеремета $M . H$. Об одном свойстве целых функций с вещественньми тейлоровскими коэффициентами // Матем. заметки. 1975. Т. 18. №3. С. 315-402.

25. Gaisin A.M. Behavior of logarithm of modulus of the sum of Dirichlet series on curves // J. Anal. 1995. V. 3. P. 205-211.

26. Гайсин А. М. Оценка ряда Дирихле с лакунами Фейера на кривых // Докл. РАН. 2000. Т. 370. №6. С. $735-737$.

27. Евграфов М. А. Об одной теореме единственности для рядов Дирихле // УМН. 1962. T. 17. №3. C. 169-175.

28. Sheremeta M. M. Five open problems in the theory of entire functions // Математичнї студії. 1996. V. 6. P. 157-159.

29. Beurling A. Some theorems on boundedness of analytic functions // Duke Math. J. 1949. V. 16. P. 355-359.

30. Hayman $W . K$. How guickly can an entire function tend to zero along curve? // Enseign Math. (2). 1978. V. 24. № 3-4. P. 215-223.

31. Евграфов М. А. Асимптотические оценки и целые функции. М.: Наука, 1979.

32. Бойчук В. С. О некоторых свойствах уточненного порядка // Сиб. матем. журн. 1979. T. 20. № 2. C. 229-236.

Институт математики

с вычислительньм центром

Уфимского научного центра

УрО РАH, г. Уфа

E-mail: gaisin@imat.rb.ru
Поступила в редакцию 14.05 .2001 и 12.05 .2003 